\title{
Self-Assembly Drugs: From Micelles to Nanomedicine
}

\author{
Paula V. Messina ${ }^{1}$, Jose Miguel Besada-Porto ${ }^{2}$ and Juan M. Ruso, \\ ${ }^{I}$ Department of Chemistry, Universidad Nacional del Sur, (8000)vv Bahia Blanca, INQUISUR-CONICET, Argentina; \\ ${ }^{2}$ Soft Matter and Molecular Biophysics Group, Faculty of Physics, University of Santiago de Compostela (USC), 15782, \\ Santiago de Compostela, Spain
}

\begin{abstract}
Self-assembly has fascinated many scientists over the past few decades. Rapid advances and widespread interest in the study of this subject has led to the synthesis of an ever-increasing number of elegant and intricate functional structures with sizes that approach nano- and mesoscopic dimensions. Today, it has grown into a mature field of modern science whose interfaces with many disciplines have provided invaluable opportunities for crossing boundaries for scientists seeking to design novel molecular materials exhibiting unusual properties, and for researchers investigating the structure and function of biomolecules. Consequently, self-assembly transcends the traditional divisional boundaries of science and represents a highly interdisciplinary field including nanotechnology and nanomedicine. Basically, self-assembly focuses on a wide range of discrete molecules or molecular assemblies and uses physical transformations to achieve its goals. In this Review, we present a comprehensive overview of the advances in the field of drug self-assembly and discuss in detail the synthesis, self-assembly behavior, and physical properties as well as applications. We refer the reader to past reviews dealing with colloidal molecules and colloidal self-assembly. In the first part, we will discuss, compare, and link the various bioinformatic procedures: Molecular Dynamics and Quantitative Structure Activity Relationship. The second section deals with the self-assembly behavior in more detail, in which we focus on several experimental techniques, selected according to the depth of knowledge obtained. The last part will review the advances in drug-protein assembly. Nature provides many examples of proteins that form their substrate binding sites by bringing together the component pieces in a process of self-assembly. We will focus in the understanding of physical properties and applications developing thereof.
\end{abstract}

Keywords: Self-assembly, Drugs, Drug-Protein Complexation, Drug delivery, Amphiphilic molecules.

\section{INTRODUCTION}

Molecular self-assembly is the process through which single molecules arrange themselves spontaneously into different structures [1]. One of the most ubiquitous selfassembly processes in physical chemistry is the hierarchical organization of amphiphile molecules into a huge variety of patterns as micelles, rods or liposomes among others [2]. Amphiphilic molecules constitute an important class of chemicals with a lot of applications in chemical process, pharmaceuticals or in food technology industries. On the other hand, they not only are highly interesting from a physicochemical viewpoint but also are fundamental to life and living bodies: it is no exaggeration to say that all living things are made up of colloids comprising a wide variety of amphiphiles[3]. Such property has recently been employed to design and fabricate for a wide range of biotechnological applications because of their relatively simple structures and easy scale up commercial productions[4]. Some drugs exhibit the same behaviour that traditional surfactants, they tend to self-aggregate, usually in a small aggregation number, when dispersed in aqueous solution in a surfactant like manner[5]. Although drug aggregates normally form at concentrations well above the concentration of the drug

\footnotetext{
*Address correspondence to this author at the Department of Applied Phsyics, Faculty of Physics, University of Santiago de Compostela, E15782, Spain; Tel: ?????????????; Fax: ?????????????; E-mail: juanm.ruso@usc.es
}

appearing in body systems, micelles may be present in the pharmaceutical formulation to overcome different challenges including poor bioavailability, stability, side effects or plasma fluctuations of drugs. Also, it is of great interest the correlation between aggregation characteristics and the clinical potency of antibiotics. The reason is that to obtain an optimal effect, the antibiotic must be administered at a low enough concentration at which aggregation is negligible. However, accidental accumulation at certain sites in the organism can possibly give rise to the formation of aggregates that do not pass through membranes; consequently, the antibiotics do not execute their therapeutic action. The association of drugs has been examined by several workers finding a change in the slope of some physicochemical properties of these drugs in aqueous solutions which are indicative of a self aggregation. This discontinuity is identified with the critical micelle concentration and the shape and size of these aggregates depend mainly on the surfactant structure, ionic strength, and temperature and so on. When the drugs are delivered through hydrogels or micelle drug carriers, the diffusion and release rates of the drug molecules may be affected. Also, the free energies for the micellization of the surface-active drug molecules in solution are of the same magnitude as the free energy for absorption of drug molecules to the carrier matrix. Hence, the hydrophobic moieties of the drug interact and tend to stabilize a cluster-like structure at the matrix of the carriers, which means that coopera- 
tive binding phenomenon has the same energetics as the micellization. Thus, surface activity of the drugs can predict their ability to cross the blood-brain barrier and can be quantified by critical micelle concentration and surface area requirement of the drug at the air/ water interface. Identification of these properties will be leading to the molecular assembly and the transport kinetics of the drug component [6].

The first studies on the aggregation characteristics of penicillins concern to penicillin $\mathrm{G}$ and correspond to work performed by Hauser et al. [7] at the Massachusetts Institute of Technology who by surface tension measurements noted that both penicillin and streptomycin salts, in the aqueous solution in which they are most commonly used for parental infections, are present in the form of colloidal micelles. This work was contradicted by Kumler and Alpen [8] who proved that this penicillin behaves as true solution and not as colloidal electrolytes. A year later, McBain et al. publish an article [9] in which show that penicillin $G$ aggregates in concentrated solution but at a critical concentration that it is of little biological interest. Studies of Hauser and Marlowe [7] and Hocking [10] set up the relationship between the activity of penicillin and their stage of aggregation. The work of Few and Schulman [11] analyzes the influence of the $\mathrm{pH}$ on the association of penicillin G. Ong and Kostenbauder report in their study [12] that micellar penicillin $\mathrm{G}$ is 2.5 times more stable than non micellar solution under the conditions of constant $\mathrm{pH}$ and ionic strength. The results of Funasaki et al. [13] permitted to know the mechanism of self-association of penicillin $\mathrm{V}$ (stepwise whithout any $\mathrm{cmc}$ ) and establish the relationship between the self-association of penicillin $\mathrm{V}$ and penicillin $\mathrm{G}$ and the effect on the bacterial activity. Thakkar and Wilham [14] using NMR techniques confirms that penicillin $\mathrm{G}$ ions aggregate in aqueous solution though hydrophobic interactions. Attwood and Argawal [15] has reported the micellar properties of several synthetic penicillins (including flucloxacillin and cloxacillin) both in water and in the presence of $0.15 \mathrm{M} \mathrm{NaCl}$. Amore recent study focused on the binary mixed system of betablocker with different lipophilicity [5]. Also the formation of monolayer of bile salts have been reported [16]. Recently, it was proposed the idea of combining two drugs forming a self-assembled system which display the drug function as well as serve as a vector [17].

On the other hand, the self-association of surface active drugs of quite different chemical structure has been widely reported [18]. Many of these drugs contain aromatic nuclei or are peptide nature. Examples of studied drugs are phenothiazine [19] and benzodiazepine [20] tranquiliziers, analgesics [21], peptide [22], antibiotics [23], tricyclic antidepressants [24], antihistamines [25], anticholinergics [26], $\beta$ blockers [5, 27], local anesthetics [28], non-steroidal antiinflammatory drugs [29], anticancer drugs [30]. Some of these structures can be observed in Fig. (1).

On the other hand, reducing costs in terms of time and material resources with computational methods have become a promising goal in Medicinal and Pharmaceutical Chemistry. There many computational techniques that can be used in this sense, to cite a few: Molecular Dynamic simulations [31], Monte Carlo methods, Docking and/or Quantitative Structure-Property Relationships (QSAR). In any case, almost all of these methods focus on few fundamental aspects including: type methods to quantify the molecular structure, type (2) methods to link the structure with the biological activity, and mathematical functions to describe the interactions and energies of the molecules.

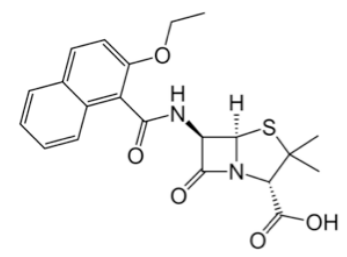

Nafcillin

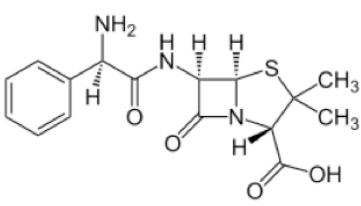

Ampicillin
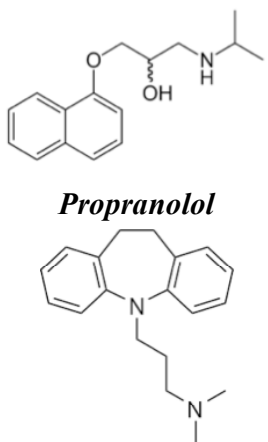

Imipramine

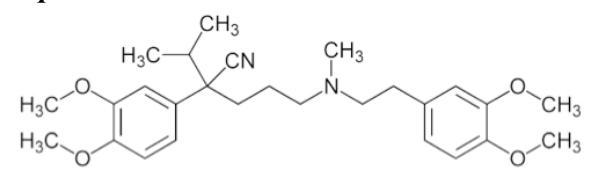

Verapamil
Fig. (1). Chemical structure of some amphiphilic drugs.

For this reasons, this review is organized as follows: there will be a short introduction on the more popular bioinformatic approaches including working principles, discussion of the specific approaches, applications, and papers that are mainly based on it. Then we have chosen some studies based on the experimental approach. Here, we include different works based on most popular and accessible techniques over the world and their interest in biotechnology and biomedical areas.

\section{MOLECULAR DYNAMIC STUDIES}

With recent increases in computing power, computer simulations have become indispensable tools. Molecular dynamic [31] simulations are probably the most popular approximation. There are two main families of MD methods, which can be distinguished according to the model chosen to represent a physical system. In the 'classical' mechanics approach to MD simulations molecules are treated as classi- 
cal objects, resembling very much the 'ball and stick' model. Atoms correspond to soft balls and elastic sticks correspond to bonds. The laws of classical mechanics define the dynamics of the system. The 'quantum' or 'first-principles' MD simulations take explicitly into account the quantum nature of the chemical bond. The electron density function for the valence electrons that determine bonding in the system is computed using quantum equations, whereas the dynamics of ions (nuclei with their inner electrons) is followed classically [32]. The overall goals of classical MD retain their original essence (chemical bonds are springs, the electron density is places only at the nucleus and is constant, atoms are soft balls) and remain focused on the predictive ability of the approach and its receptiveness to physical interpretation. In molecular dynamics, the forces between molecules are calculated explicitly and the motion of the molecules is calculated by means of a numerical integration method on a computer, basically solving Newton`s equation of motion for the atoms of the system. The initial conditions are the positions of the atoms and their velocities. From the initial positions, velocities and forces, it is possible to calculate the positions and velocities of the atoms at a small time interval, time step (usually of the order of 1 femtosecond), later. The cycle has to be repeated many times in the course of a full simulation, usually for many thousands of time steps. The forces are almost always based on an empirical potential such as the Lennard-Jones potential, electrostatic. A great advantage of the molecular dynamics method, is that it explicitly describes the molecular system as a function of time, this means it can directly calculate time dependent phenomena. The molecular dynamics method was first introduced by Alder and Wainwright in the 50's to study the interactions of hard spheres (atoms that interact through perfect collisions) [33]. One decade later, Rahman performed the first simulation using a realistic potential for liquid argon [34]. At the turn of the 70's the first protein simulations appeared. Most authors claim that the first publication correspond to the simulation of the bovine pancreatic trypsin inhibitor (BPTI) performed by McCammon, when he was a research fellow at Harvard collaborating with Martin Karplus [35]. The simulated protein was the small bovine pancreatic trypsin inhibitor (BPTI), with approximately 500 atoms, in vacuum, and the simulation time was only 9.2 ps. However, is it also proposed the work of Levitt et al. [36]. The first simulation concerning to macromolecule-ligand interactions was developed by Case et al. in 1979 [37]. Three years later Kuntz et al. marks the beginning of the problem of "docking" molecules to each other: DOCK [38]. Here arises the pharmaceutical computation industry. By the same time Sundaram et al. reported molecular dynamic simulation of the drug norepiniphrine, demonstrating the possible ways of superposing a phenyl ring of a tricyclic antidepressant on the phenyl ring of norepinephrine [39]. Jonsson et al. reported molecular dynamics simulations of a sodium octanoate micelle in aqueous solution which results agree perfectly with NMR measurements [40]. In 1988 the first simulation of a protein in explicit water is carried [41]. In 1990 the GROMOS force field is applied in the simulation of the complexation between deoxyninucleoside and proflavin [42]. One year later, MD simulations of the protein-inhibitor (chicken dihydrofolate reductase-trimethoprim) complexes, and the evaluation of potential energy interactions between inhibitor and protein, were evaluated for their ability to predict trends similar to those found for the thermodynamic simulations [43]. Next year, Heimstad et al. reveals the molecular structure, electrostatic potentials and dynamics of imipramine, chlorimipramine, amitriptyline and nortriptyline, using the AMBER all atom force field [44]. In $2001 \mathrm{Kim}$ et al. revealed that the size of chitosan self-aggregates varied in the range. of 130300 run in diameter, and their structures were found to depend strongly on the molecular weight of chitosan ranging from 5 to $200 \mathrm{kDa}$ [45]. Meanwhile, in other interesting work MD was employed to elucidate the molecular details of the process of fusion for small lipid vesicles. The simulations are based on a coarse grained (CG) lipid model that accurately represents the lamellar state of a variety of phospholipids and enables us to observe intermediate stages during fusion at near atomic detail [46]. The review of Alonso et al. in 2006, focusing on applications and protocols of recent studies where docking calculations and molecular dynamics [31] simulations were combined to dock small molecules into protein receptors, is also remarkable. Recently, the MD simulations of the drug benzothiazole were performed to characterize the structure and dynamic behavior of the corresponding aggregates at several concentrations of the drug [30]. Also the structure and properties of the structures spontaneously formed within aqueous mixtures of the hydrogenated/fluorinated catanionic surfactant cetyltrimetylammonium perfluorooctanoate in the absence of counterions as a function of its concentration were investigated by computational study at room temperature [2b].

\section{QUANTITATIVE STRUCTURE ACTIVITY RELA- TIONSHIP (QSAR) STUDIES}

It has been nearly 40 years since the quantitative structure-activity relationship (QSAR) paradigm first found its way into the practice of agrochemistry, pharmaceutical chemistry, toxicology, and eventually most facets of chemistry. Its staying power may be attributed to the strength of its initial postulate that activity was a function of structure as described by electronic attributes, hydrophobicity, and steric properties as well as the rapid and extensive development in methodologies and computational techniques that have ensued to delineate and refine the many variables and approaches that define the paradigm [47]. QSAR studies can reduce the costly failures of drug candidates in clinical trials by filtering the combinatorial libraries. Virtual filtering can eliminate compounds with predicted toxic of poor pharmacokinetic properties early in the pipeline. The chemoinformatic methods used in building QSAR models can be divided into three groups, i.e., extracting descriptors from molecular structure, choosing those informative in the context of the analyzed activity, and, finally, using the values of the descriptors as independent variables to define a mapping that correlates them with the activity in question [48]

In 1996 Huibers et al. [49] propose a general threeparameter structure -property relationship was developed for a diverse set of 77 nonionic surfactants, employing topological descriptors calculated for the hydrophobic fragment of the surfactant molecule. The three descriptors represent contributions from the size of the hydrophobic group, the size of the hydrophilic group, and the structural complexity of the hydrophobic group. They found that the cmc can be pre- 
dicted for a diverse set of nonionic surfactants using the equation

$$
\begin{aligned}
& \log _{10} c m c=-(1.80 \pm 0.16)-(0.567 \pm 0.009) c-K H O+ \\
& (1.054 \pm 0.048) c-A I C 2+(7.5 \pm 1.0) R N N O
\end{aligned}
$$

The Kier \& Hall index of 0 order for the hydrophobic fragment (c-KH0) represents the size of the hydrophobic fragment and contains group contributions from all nonhydrogen atoms in the fragment. The average information content of order 2 for the hydrophobic fragment (c-AIC2) represents the complexity of the hydrophobic fragment. The final descriptor, the relative number of nitrogen and oxygen atoms (RNNO), represents the size of the hydrophilic fragment.

The aggregation properties of different amphiphilic antimicrobially active 2,2'-bipyridyl monoammonium salts were studied by means of QSAR, the authors discovered that from quantitative dependencies between lipophilicity (expressed by means critical concentration of micelle formation), structure (length of alkyl chain) and antimicrobial activity (minimum inhibition concentration) that the maximum of activity is achieved with compounds of chain length $\mathrm{m}=$ 13 to $16[50]$.

Jalali-Heravi et al. [51] related the cmc of a set of 51 alkylpoyoxiethylene glycol ethers and alkylphenol ethers to topological, electronic and molecular structure parameters. Topological descriptors include Winer number, Tandic and Balaban indexes. Geometric descriptors include van der Waals volume of the molecule, maximum distance between the atoms in the molecule, surface area of the head and tail of the molecules. Electronic descriptors consist of heat of formation and dipole moments of the molecules. From the obtained results it was concluded that geometric parameter and van der Waals interactions play a major role in selfaggregation of nonionic amphiphiles.

The critical micelle concentration $(\mathrm{cmc})$ values of a 49surfactant dataset derived from lactic, malic, and tartaric acids and from gluconolactone were subjected to Quantitative Structure Property Relationship (QSPR) studies [52]. The model obtained takes into account seven descriptors. Five of them are topological and depend on the twodimensional structure of surfactants: Balaban index (JX), First kappa shape index (Kappa-3), Kier molecular flexibility index (PHI), second-order atomic connectivity index (CHI2 ), and Wiener index. Authors also identified the octanolwater partition coefficient and the dipole moment as suitable measures of the cmc. Finally, the model used was as follows:

$$
\begin{aligned}
& \log _{10} c m c=5.868+1.175 J X-0.364 \text { Kappa }- \\
& 3-0.166 \text { PHI }-0.482 \text { CHI }-2+0.00128 \text { Wiener }[3]+ \\
& +0.171 \text { Dipole }- \text { mag }+0.203 \log P
\end{aligned}
$$

In 2006, the Katritzky et al. [53] investigate a data set of 119 diverse anionic surfactants to relate the logarithm of critical micelle concentration (cmc) to the molecular structure using Comprehensive Descriptors for Structural and Statistical Analysis (CODESSA Pro) software. The regression equation obtained contained variables that include features of both hydrophobic and hydrophilic fragments of the surfactant:

$$
\begin{aligned}
& \log _{10} c m c=(1.89 \pm 0.11)-(0.314 \pm 0.010) t \\
& - \text { sumKier \& Hall index }(0 \text { th order })- \\
& -(0.034 \pm 0.003) \text { total dipole of the molecule }- \\
& -(1.45 \pm 0.18) h-\text { sum }- \text { relative number of carbon atoms }
\end{aligned}
$$

In this equation the t-sum-Kier \& Hall index of 0 th order is the sum of the Kier \& Hall index of zeroth order over all hydrophobic tails; the total dipole of the molecule is the AM1 calculated total dipole moment of the molecule; and the h-sum relative number of carbon atoms is the sum of the relative number of carbon atoms over all hydrophilic heads. A large number of molecular descriptors were explored in the initial descriptor pool, but the cmc was proved to be appropriately modeled by the use of fragment descriptors defined for the hydrophobic and hydrophilic domain of the molecule.

In other interesting work, the developed QSARs provide insight into the mechanism of cytotoxic action of antimicrobial peptides. In a subsequent blind test, the QSAR correctly ranked four of five protegrin analogues newly synthesized and tested for toxicity [54].

Later, Katritzy et al. [55] developed Linear and nonlinear predictive models for 50 ammonium and quaternary pyridinium cationic surfactants for both the first and second critical micelle concentrations. For the first cmc they found five descriptors. The descriptors were classified as follows: topological-Complementary Information Content (order 0) (D1), Average Information Content (order 0) (D3) and Wiener index (D4), charge-related FPSA-3 Fractional PPSA (PPSA-3/TMSA) [Semi-MO PC] (D2), and quantum chemical-minimum electron-nuclear attraction for a $\mathrm{C}-\mathrm{N}$ bond (D5). The significance of these descriptors of the model according to $t$-test criterion was: D1 $>$ D2 $>$ D4 $>$ D3 $>$ D5 . The experimental data points available for the second CMC resulted in a model including just three descriptors. The most significant descriptor in the three-parameter model is the surface weighted charged partial positive surface area WPSA-3 Weighted PPSA(PPSA2*TMSA/1000)[Semi MO PC]. The WPSA-3 Weighted PPSA (PPSA$3 *$ TMSA) $/ 1000[$ Semi-MO PC] is a fragment descriptor calculated for the hydrophobic domain. The second one, the minimum exchange energy for a $\mathrm{C}-\mathrm{C}$ bond descriptor is defined for the hydrophilic domain of the surfactant molecule. This energy reflects the change in the Fermi correlation energy between the two electrons localized on $\mathrm{C}$ atoms and describes the conformational changes in the hydrophobic heads of the surfactant that play an important role in the aggregation process. The last descriptor, the relative positive charge (QMPOS/QTPLUS)[Semi MO PC] is defined for the hydrophobic domain of the surfactant. The charge distribution in the surfactant molecule plays an important role in the aggregation of cationic surfactants. This descriptor has a large negative influence in the equation that is in accord with previous quantum chemical calculations that states that $\mathrm{CMC}$ increase when the surfactant tail gains positive charge, respectively, the solubility of the molecule increase.

MARCH-INSIDE (Markov Chain Invariants for Networks Simulation and Design) is a well-known type method for QSAR analysis. Recently, González-Díaz et al. [56] have 
combined MI with ANNs (Artificial Neural Networks) in order to seek QSAR models. The name of this strategy is MIANN (MARCH-INSIDE \& ANN models). The MARCHINSIDE approach [57] is based on the calculation of the different physicochemical molecular properties as an average of atomic properties $\left(\mathrm{w}_{\mathrm{j}}\right)$. For instance, it is possible to derive average estimations from molecular descriptors or local indices such as electronegativity values ${ }^{\mathrm{k}} \chi(\mathrm{G})$ [58].

$$
\begin{gathered}
{ }^{k} \chi(G)=\sum_{j \in G} p_{k}\left(\chi_{j}\right) \cdot \chi_{j} \\
\theta_{k}(G)=-\sum_{j \in R}^{n}{ }^{k} p_{j}(G) \cdot \log \left[{ }^{k} p_{j}(G)\right] \\
\pi_{k}(G)=\sum_{i=j \in R}^{n}{ }^{k} p_{i j}(G)
\end{gathered}
$$

In this case, $\mathrm{w}_{\mathrm{j}}=\chi_{\mathrm{j}}$, the atomic electronegativity. It is possible to consider isolated atoms $(k=0)$ in a first estimation of the molecular properties ${ }^{0} \chi(\mathrm{G}),{ }^{0} \theta(\mathrm{G})$, or ${ }^{0} \pi(\mathrm{G})$. In this case, the probabilities ${ }^{0} \mathrm{p}\left(\chi_{\mathrm{j}}\right)$ are determined without considering the formation of chemical bonds (simple additive scheme). However, it is possible to consider the gradual effects of the neighboring atoms at different distances in the molecular backbone. In order to reach this goal, the method uses an MCM, which determines the absolute probabilities $\mathrm{p}_{\mathrm{k}}\left(\mathrm{w}_{\mathrm{j}}\right)$ with which the atoms placed at different distances $k$ affect the contribution of the atom $j$ to the molecular property in question. In that work Ref humberto [56] authors obtained by the first time a linear model able to predict the probability which with the compound (i-th) presents an experimental value of the property ( $\mathrm{j}$-th) higher than the average value of this property for this data set. The best model found using LDA was the following:

$$
\begin{aligned}
S_{i}\left(Z_{i j}>0\right)= & 3,91375+0,011557 \cdot e^{C_{\text {sal }}\left(m o l \cdot L^{-1}\right)}-0,012303 \cdot T(K)_{j} \\
& +0,244894 \cdot{ }^{5} \chi\left(\text { anion }_{i}-0,047320 \cdot{ }^{5} \chi(\text { cation })_{i}\right. \\
& +1,919078 \cdot{ }^{5}\langle\chi\rangle\left(\text { anions }_{j}\right)+3,436077 \cdot{ }^{5}\langle\chi\rangle(\text { cations })_{j} \\
& n=1085 \quad \chi^{2}=221,2429 \quad p\left(\chi^{2}\right)<0.01
\end{aligned}
$$

In this equation, $\mathrm{S}_{\mathrm{ij}}\left(\mathrm{Z}_{\mathrm{ij}}>0\right)$ is a real-valued score (output of the linear model) that can be used to discriminate between surfactans with high probability $\mathrm{p}\left(Z_{\mathrm{ij}}>0\right)$ of showing a high $Z_{\mathrm{ij}}>0$ and others with high probability $\mathrm{p}\left(Z_{\mathrm{ij}} \leq 0\right)$ of presenting a $Z_{i j} \leq 0$. Considering that $Z_{i j}$ is an standardization coefficient used to scale the values of the different properties to a single adimensional scale. We calculated this parameter as follow: $Z_{i j}=\left(Y_{i j}-\left\langle Y>_{j}\right) / S D_{j}\right.$. Where, $Y_{i j}$ is the value of the $j-$ th property of the $\mathrm{i}$-th surfactant; $\langle\mathrm{Y}\rangle_{\mathrm{j}}$ is the average value of his property in the data set and $\mathrm{SD}_{\mathrm{j}}$ the standard deviation. These parameters allowed us to classify the surfactants as "active" $=>\mathrm{C}=1 \Rightarrow \mathrm{Z}_{\mathrm{ij}}>0=>\mathrm{Y}_{\mathrm{ij}}><\mathrm{Y}>_{\mathrm{j}}$ or "non-active" $=>\mathrm{C}=-1=>\mathrm{Z}_{\mathrm{ij}} \leq 0=>\mathrm{Y}_{\mathrm{ij}} \leq<\mathrm{Y}>_{\mathrm{j}}$. It means, surfactants with a higher or lower-than-the-average value of the observed property $\mathrm{j}$-th. The independent terms of the equation are: $\mathrm{C}_{\text {salt }}$ is the concentration of the salt in the solvent used; $\chi$ is the average electronegativity of the surfactant for the anion or cation part; and $\langle\chi\rangle$ are the average value of $\chi$ for those surfactants with $Z_{i j}>0$. This terms were calculated using the software MARCH-INSIDE. In addition, $\mathrm{n}$ is the number of cases used to train the model and $\chi^{2}$ is Chi-square statistics with a given $p$-lvel $=p\left(\chi^{2}\right)$. They used this linear model to predict satisfactorily the self-aggregation behavior of imipramine hydrochloride in other experimental conditions different from those used in the previous experiments.

\section{SELF-AGGREGATION IN DRUG SYSTEMS}

The solubility of amphiphilic drugs in water is limited and produce a local ordering of the water molecules that surround the hydrophobic moiety, that is, the hydrated head groups can take part in the H-bonding structure of the water producing the formation of micelles or other type of aggregates. In addition, this spontaneous process is governed by the hydrophobic effect, which is related with the fact that the contact between water molecules and non-polar moieties of the drug molecule are minimized and the entropy of the system is increased. Furthermore, there is an additional enthalpic term associated to the van der Waals clustering of the chains. Micelle formation is a cooperative process that occurs over a narrow range of concentration and also over a critical temperature (called Krafft point), where the transition from the monomeric solution to a solution containing both monomers and micelles takes place. For that reason, it is necessary to define a concentration within this narrow range as critical micelle concentration ( $\mathrm{cmc}$ ) that is considered the saturation concentration for monomers. Furthermore, it is worth mentioning the strong dependence of the $\mathrm{cmc}$ on the chemical structure of the drug, the temperature and also on the presence of different cosolutes such as electrolytes or alcohols. A successive rise of drug concentration leads to an increase in the number of micellar aggregates, prior to any growth of the aggregates.

Micelles may be characterized by the aggregation number $\left(N_{\text {agg }}\right)$, and the molecular length sets the maximum radius for the spherical micelle shape. According to these considerations, a packing parameter $P$ is defined as the ratio between the volume $v$ of the hydrophobic moiety of the molecule and the product of the area occupied by the head group (polar group) $a$ with the length $l$ of the hydrophobic moiety of the molecule: $P=v / a l$. The values of the packing parameter give us an idea about the type of structure: spherical micelles $(P<1 / 3)$, elongated micelles $(P<1 / 2)$, vesicles $(P<$ $1)$ and lamellae $(P \approx 1)$. Additional factors that affect the micelle shape are the optimal head group area, the volume and length of the hydropobic moiety. The net aggregation of an ionic micelle is found to be less than the degree of micellar aggregation. This indicates that large fractions of counterions remain associated with the micelle. These counterions form the Stern layer at the micellar surface. In ionic micelles, the Stern layer, which resembles a concentrated electrolyte solution, consist of bound ionic surfactant head groups, counterions and water molecules. The water is present as both free molecules and water of hydration. The layer just beyond the Stern layer is a diffuse layer, known as the GouyChampman layer, extending outward to several hundred angstroms.

\subsection{Thermodynamics of Self-Aggregation}

Thermodynamic costs indicate whether processes are likely to occur and are conveniently quantified in terms of a free energy $\Delta \mathrm{G}$. In the context of solvating a molecule, $\Delta \mathrm{G}$ is the reversible work for the solvent to reorganize and solvate 
the solute. The thermodynamics of micellization is based on the Gibbs-Helmholtz equation: $\Delta G_{m}=\Delta H_{m}-T \Delta S_{m}$, where $\Delta H_{m}$ and $\Delta S_{m}$ are the enthalpic and entropic changes incurred during solvation. The enthalpic part is a measure of the average potential energy of interaction between molecules, and the entropic part is a measure of the order or intermolecular correlations. The free energy of a process involving significant changes in the number of molecular interactions, such as the breaking of hydrogen bonds to form a liquid-vapour interface, will be dominated by its enthalpic component [59].

A process that requires specific spatial organization of hydrogen bonding patterns will have an important entropic component. At room temperature, for instance, the entropic cost of hydrating small hydrophobic species is dominant, as manifested by $\Delta G$ increasing with increasing temperature. With a sufficient increase in temperature, however, the extent of hydrogen bonding between water molecules diminishes, and maintaining hydrogen bonds becomes less important. In fact, whereas the entropy change associated with the hydration of a small alkane at room temperature is negative (and reasonably large in magnitude), it becomes positive near the boiling temperature of water [60].

Aggregates or micelles are dynamic species; in fact there is a constant, rapid interchange (typically on a microsecond timescale) of molecules between the aggregate and the solution pseudo-phases. This constant formation-dissociation process relies on a subtle balance of interactions. These interactions come from contacts between: hydrocarbon chainwater, hydrocarbon-hydrocarbon chains, head group-head group, and from salvation of the head group. There, the net free energy change upon micellization, $\Delta G_{m}$, can be written as:

\section{$\Delta G_{m}=\Delta G(H C)+\Delta G($ contact $)+\Delta G($ packing $)+\Delta G(H G)$}

where $\Delta G(H C)$ is the free energy associated with transferring hydrocarbon chains out of a water and into the interior of the micelle; $\Delta G$ (contact) is the free energy attributed to solvent-hydrocarbon contacts in the micelle. $\Delta G$ (packing) is a positive contribution associated with confining the hydrocarbon chain to the micelle core; and $\Delta G(H G)$ is a positive contribution associated with head group interaction, including electrostatic as well as head group conformation effects. Aggregation of drug molecules results from the tendency of the hydrophobic groups to minimize contacts with water by forming oily microdomains with the solvent. There, interactions among non charged moieties are maximized, while hydrophilic head groups remain surrounded by water. The predominant effect of the drug molecule is to increase the degree of structure in the immediately surrounding water. This is one of the main features of the hydrophobic effect, which was studied in detail by Tanford to account the very light solubility of hydrocarbons in water [61]. During the micellar aggregation the reverse process occurs, that is, as lyophobic residues aggregate, the highly structured water around each chain collapses back to ordinary bulk water thereby accounting for the apparent large overall gain in entropy, $\Delta S_{m}$.

The mechanism of micelle clustering process from surfactant monomers, S, can be described by a series of stepwise equilibrium

$$
S+S \stackrel{K_{2}}{\leftrightarrow} S_{2}+S \stackrel{K_{3}}{\leftrightarrow} S_{3}+\ldots \stackrel{\kappa_{n}}{\leftrightarrow} S_{n}+S
$$

where the thermodynamic parameters for the aggregation process can be expressed in terms of equilibrium constants $K_{\mathrm{n}}$. However, each $K_{\mathrm{n}}$, cannot be measured individually, so different approaches have been proposed to model the energetic of the process of self-assembly. Although not totally accurate, two simple models are generally encountered: the mass action model and the phase separation model. In the mass action model, with the size range of spherical micelles around the cmc being very limited, it is assumed that only one of $K_{\mathrm{n}}$ value is dominant, and micelles and monomeric species are considered to be in chemical equilibrium:

$$
n S \leftrightarrow S_{n}
$$

where $n$ is the number of drug molecules, $S$, associating to form a micelle [62], that is, the aggregation number.

Considering only univalent counterions, it is possible to describe for example ionic surfactants as $M^{+} S^{-}$and assume these are completely dissociated into $M^{+}$and $S$. The selfassembly of low molecular weight anionic surfactant molecules to form micelles may be represented by

$$
n S^{-}+m M^{+} \leftrightarrow\left(S_{n}^{-} M_{m}^{+}\right)^{z-}
$$

where $n$ is the degree of aggregation and $z$ is the net charge of the micelle which is given by $z=n-m$. The charge of the micelle may also be expressed as the fraction ionized, $\alpha=(n-m) / n$.

The chemical equilibrium between monomeric drug ions and micelles is assumed with a corresponding equilibrium constant, $K_{\mathrm{n}}$, and in mass action approach is given by:

$$
K=\frac{\left[a_{\text {mic }}\right]}{\left[a_{S}\right]^{n}\left[a_{M}\right]^{m}}
$$

where the brackets indicate the activities of the indicated species.

The equilibrium constant gives the standard free energy of micellization

$$
\Delta G^{0}=-R T \ln K=-R T\left(\ln a_{m i c}-n \ln a_{S}-m \ln a_{M}\right)
$$

The standard free energy per mole of drug evaluated at the cmc $\left(a_{M} \approx a_{S}=a_{c m c}\right)$ becomes:

$$
\Delta G_{m i c}^{0}=-R T\left[(1+m / n) \ln a_{c m c}-\frac{1}{n} \ln a_{m i c}\right]
$$

where $m / n \equiv \beta$ represents the fraction of charges of micellized univalent drug ions neutralized by micelle-bound univalent counterions. Assuming that the aggregation numbers is large $(>100)$ we can do an approximation on the expression of $\Delta G^{o}{ }_{m i c}$ :

$$
\Delta G_{m i c}^{0} \approx R T(1+m / n) \ln a_{c m c}
$$

Considering that cmc occurs in dilute solutions, so activity may be replaced by the concentration of the drug at the cmc

$$
\Delta G_{m i c}^{0} \approx R T(1+m / n) \ln c m c
$$




\subsection{External Factors Affecting the Self-Aggregation}

The tendency toward aggregation process may be strongly affected by the structure of the drug, the counterion nature, the presence of additives or the change in temperature $[19,27 \mathrm{~d}, 27 \mathrm{~g}, 63]$. The length of the hydrophobic moiety is a major factor determining the cmc. As an example, for a homologous series of linear single chain surfactants, the cmc decreases logarithmically with carbon numbers. The relationship usually fits the Klevens equation [64]: $\log (\mathrm{cmc})$ $=A-B n_{c}$. Where $A$ and $B$ are constants for a particular homologous series and temperature, and $n_{c}$ is the number of carbon atoms in the chain. The constant $A$ varies with the nature and number of hydrophilic groups.

Variations in the hydrophilic head group affect the cmc. In general, drug containing ionic head group have a higher cmc than those containing nonionic head groups. This is due to electrostatic repulsion between the head groups of neighboring molecules within the micelles. A polar group reduces the overall hydrophobicity of an apolar solute by an amount equivalent to several methylene groups: the hydration shells of polar groups overlap with those of methylene groups. Alternatively, introduction of polar functionalities increases the solubility of apolar solutes since it reduces the necessity for creating a complete hydrophobic hydration shell. Generally, hydration of the polar group extends to the third carbon atom in the apolar moiety of drug molecules [27d, e, 65].

The counterion atmosphere surrounding the micelles leads to preferential counterion binding that affects the shape, size and interaction on the micelles. Ionic surfactants may form a variety of micellar structures depending on the nature of the counterion. The smaller and more hydrophilic counterions induce formation of spherical micelles. This is due to the weak binding of these counterions to the micelles which leads to an effective decrease of the electrostatic repulsion between the drug head groups. Ionic drug with organic counterions form thread-like structures that result in viscoelastic behavior of the solution [66]. This effect is attributed to the much stronger binding of the counterions to the micelles and even to intercalation between oppositely charges surfactant head groups. As a result, the electrostatic repulsion between the drug head groups is screened more effectively which reduces the curvature of the surfactant spherical aggregate and lead to formation of cylindrical micelles.

The presence of electrolytes causes a decrease in the cmc of most surfactants [27f, 67], that is, when ions are added to a micellar solution, can dehydrate the surfactant headgroups. This causes an increase of the structural packing parameter and as a consecuence, the $\mathrm{cmc}$ is reduced and the aggregate morphology changed. The effects are caused by destruction of the hydration layer of the drug, decreased electrostatic repulsions, and a increased counterion binding. The greatest effect is found for ionic drugs. The main effect of the salt is to partially screen the electrostatic repulsion between the head groups and so lower the $\mathrm{cmc}$.

\subsection{Effect of Temperature}

The temperature dependence of the cmc of most ionic surfactants describes a U-shape curve, as it is shown in Fig. (2). This behavior is interpreted as a consequence of two opposing effects: the hydration of the head group and the structured water molecules surrounding the hydrophobic moiety. The increase in temperature produces a decrease in the hydration of the head group and an increase in the breakdown of the structured water molecules surrounding the hydrophobic part. The first favors micelle formation while the second does not. The first effect is dominant at lower temperatures and the second at higher, so the minimum $\left(T^{*}\right.$ and $\left.c m c^{*}\right)$ represents the compensation of both effects. The second effect becomes greater when the hydrophobic moiety length increases. The decrease in the minimum when the hydrophobicity increases is consistent with this argument.

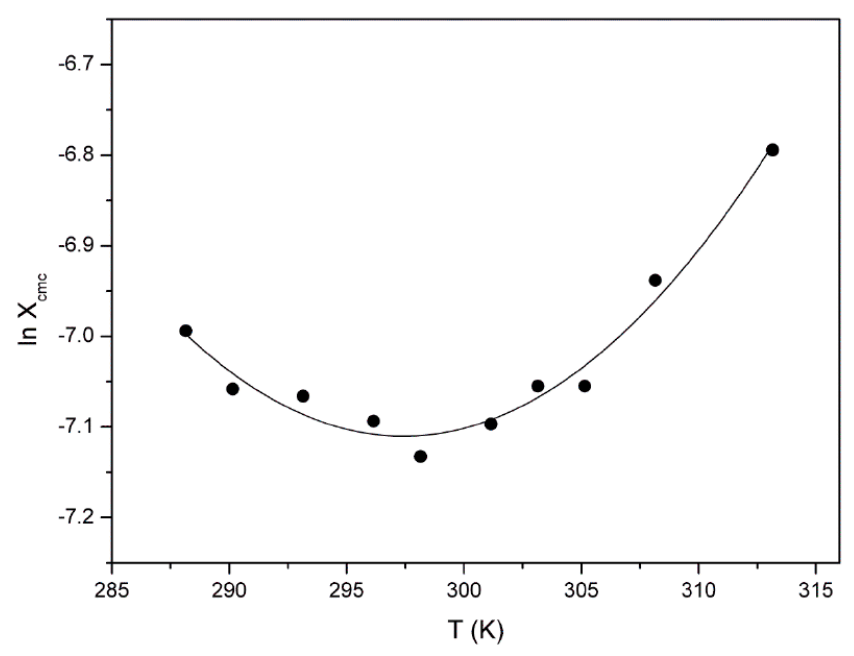

Fig. (2). Natural logarithm of the critical aggregation concentration as a function of temperature. The solid line corresponds to the fit of experimental data to the thermodynamic model proposed by Rodriguez et al.

A first analysis of the relations between cmc and temperature was developed by La Mesa [68]. He assumed that $c m c_{\text {min }}$ and $T_{\min }$ are reference values for the micellization, so it is possible to fit experimental data to the equation

$$
\mathrm{cmc} / \mathrm{cmc} *-1=|1-T / T *|^{\gamma}
$$

where $\gamma$ is an exponent whose numerical value is $1.74 \pm$ 0.03 without physical meaning. Although La Mesa used his model to forecast $\mathrm{cmc}$ values in a wide range of experimental conditions and with different compounds, it have some problems with some systems [69]. Later, Muller [70] described the variation of $\mathrm{cmc}$ with temperature by means of the equation

$$
\ln \left(c m c / c m c^{*}\right)=\left\{\Delta C_{p} /(1+\beta) R\right\}\left\{1-T^{*} / T+\ln (T * / T)\right\}
$$

where $\Delta C_{p}$ is the heat capacity and $\beta$ the degree of ionization. Although in this equation variables with physical meaning are introduced, the preliminary assumptions $\left(\Delta C_{p}\right.$ and $\beta$ constant with temperature) are quite severe.

Kang et al. [71] proposed a simple modification of $\mathrm{La}$ Mesa's equation, introducing a constant:

$$
c m c / c m c *-1=A|1-T / T *|^{B}
$$


These authors have also proposed an elegant description of the cmc as a function of temperature; however, it is possible that the excessive number of variables in their model has forced them to introduce too severe approximations. For example, the compensation temperature and the temperature at which entropy becomes zero have constant values for most surfactants, but it is possible to find references in the literature that show that these values depend on the molecule [72] and even depend on the electrolyte concentration [27e]. Assuming a linear behavior of enthalpy of aggregation with temperature, the slight dependence of $\beta$ with temperature that in some cases could be assumed as a constant and taking into account the linear behavior of the enthalpy-entropy compensation phenomena, the relation is as follows:

$$
\ln x^{*}=A+B T+\frac{C}{T}-\frac{1}{(2-\beta) n} \ln \left[2 n^{2}(2-\beta)\right]-\ln \frac{2}{v_{S}}
$$
$C$ are

where $v_{\mathrm{S}}$ is the valence of the surfactant ion and $A, B$ and

$$
\begin{gathered}
A=\frac{\Delta C_{P, m}^{0}}{(2-\beta) R}\left(1+\frac{T_{S=0}}{T_{C}}\right)<0 \\
B=\frac{-\Delta C_{P, m}^{0}}{(2-\beta) R T_{C}}>0 \\
C=\frac{-\Delta C_{P, m}^{0} T_{H=0}}{(2-\beta) R}>0
\end{gathered}
$$

where, $T_{H=0}, T_{S=0}$ and $T_{C}$ are the temperature at $\Delta H^{o}{ }_{m}=0$, $\Delta S^{o}{ }_{m}=0$ and the compensation temperature, respectively. The logarithmic terms can be neglected because usually they are much lower than the first three terms. In the final equation, $\beta$ and $\Delta C_{p, m}^{o}$, and $T_{H=0}, T_{S=0}$ and $T_{C}$ are considered as constants. A known constant value can be $T_{C}$, known as compensation temperature, and the same is assumed for all the ionic molecules. Furthermore, $\beta$ can be used as a known constant and equal to $\beta$ at $T^{*}$. Using these parameters and taking $T_{H=0}, T_{S=0}$ and $\Delta C_{p, m}^{o}$ as free parameters, all the relevant thermodynamic parameters of the aggregation process can be obtained

Recently, Rodriguez et al. [73] improved Muller's equation by assuming that the degree of ionization of the aggregates increases linearly with temperature, $\beta=\beta_{0}+\beta_{1} T$, and the standard change in heat capacity varies linearly in the temperature range studied by means of the equation

$$
\Delta C_{P, m}^{0}=\Delta C_{P, m}^{0^{*}}+\alpha\left(T-T^{*}\right)
$$

Thus, based on these assumptions they arrived at the following equation:

$$
\begin{aligned}
& \ln x_{c m c}=\ln x_{c m c}^{* *}\left\{1+\beta_{1} T^{*}\left[\frac{1}{2-\beta}\left(\frac{T^{*}}{T}-2-\frac{\beta_{0}-2}{\beta_{1} T^{*}}\right)+\frac{1}{2-\beta^{*}}\left(1+\frac{\beta_{0}-2}{\beta_{1} T^{*}}\right)\right]\right\}+ \\
& +\frac{\Delta C_{P, m}^{0^{*}}}{(2-\beta) R}\left(1-\frac{T^{*}}{T}-\ln \frac{T}{T^{*}}\right)+\frac{\alpha}{(2-\beta) R}\left(\frac{T^{* 2}-T^{2}}{2 T}+T^{*} \ln \frac{T}{T^{*}}\right)
\end{aligned}
$$

This equation relates entropy, enthalpy and heat capacity of aggregation with the corresponding Gibbs free energy as a function of temperature. The parameters in this equation are: $T^{*}, \ln x_{c m c}, \Delta C^{o *}{ }_{p, m}, \alpha, \beta_{0}$ and $\beta_{1}$. At a minimum, $\Delta H_{m}^{o}$ and $\Delta S^{o}{ }_{m}$ can be obtained from the following relations:

$$
\begin{gathered}
\Delta H_{m}^{0^{*}}=R T^{* 2} \beta_{1} \ln x_{c m c}^{*} \\
\Delta S_{m}^{0^{*}}=\frac{\Delta H_{m}^{0^{*}}}{T^{*}}\left(2+\frac{\beta_{0}-2}{\beta_{1} T^{*}}\right)
\end{gathered}
$$

In some cases the temperature dependence of $\beta$ is not evident and can be taken as a constant. An appropriate equation was recently used by González-Pérez et al. [74]

$$
\begin{aligned}
& \ln x_{c m c}=\ln x_{c m c}^{*}+\frac{\Delta C_{P, m}^{0^{*}}}{(2-\beta) R}\left(1-\frac{T^{*}}{T}-\ln \frac{T}{T^{*}}\right)+ \\
& \frac{\alpha}{(2-\beta) R}\left(\frac{T^{* 2}-T^{2}}{2 T}+T^{*} \ln \frac{T}{T^{*}}\right)
\end{aligned}
$$

The parameters in this equation are: $T^{*}, \ln x_{c m c}, \Delta C^{o *}{ }_{p, m}$, $\alpha, \beta$, and in this case $\Delta H^{o}{ }_{m}$ and $\Delta S^{o}{ }_{m}$ remain as follows:

$$
\begin{gathered}
\Delta H_{m}^{0^{*}}=0 \\
\Delta S_{m}^{0^{*}}=R \ln x_{c m c}^{*}(\beta-2)
\end{gathered}
$$

Another possibility can be developed by taking into account the linear dependence of $\mathrm{b}$ with temperature, letting $\Delta C^{o *}{ }_{p, m}$ remain constant with temperature. This possibility was used recently by González-Pérez et al. [75]. The expression remains as follows:

$$
\begin{aligned}
& \ln x_{c m c}=\ln x_{c m c}^{*}\left\{1+\beta_{1} T^{*}\left(\frac{1}{2-\beta}\left(\frac{T^{*}}{T}-2-\frac{\beta_{0}-2}{\beta_{1} T^{*}}\right)+\frac{1}{2-\beta^{*}}\left(1+\frac{\beta_{0}-2}{\beta_{1} T^{*}}\right)\right]\right\}+ \\
& +\frac{\Delta C_{P, m}^{C^{*}}}{(2-\beta) R}\left(1-\frac{T^{*}}{T}-\ln \frac{T}{T^{*}}\right)
\end{aligned}
$$

We report now the thermodynamic parameter of aggregation for several penicillins: sodium cloxacillin, sodium dicloxacillin, nafcillin sodium, penicillin V [23b], ampicillin sodium [23a], oxacillin sodium [76] and penicillin G [72]. First of all we have to divide these penicillins in two groups as a function of their present one or two stage self association process. The first group will be constitute by cloxacillin, dicloxacillin and nafcillin, meanwhile penicillin $\mathrm{G}$ and $\mathrm{V}$, ampicillin and oxacillin will be in the second one. In two stage self association process, an initial association occurring in dilute solution, leads to the formation of small aggregates which may then self associate to form larger aggregates at a second critical concentration, corresponding to the reported cmc. Obviously thermodynamic parameters will be calculated in every stage. The variation of the cmc with temperature for these penicillins can be fitted to any of the thermodynamic models previously proposed. In all cases, the curve passes through a minimum around $300 \mathrm{~K}$, except for ampicillin at $295 \mathrm{~K}$. Penicillins with two stage association have showed the same minimum for both stages. An interpretation of these variation has been suggested by Zielinsky [77] postulating the existence of two hydrations around the amphiphile molecule, one around the hydrophobic part and other around the polar headgroup. The increase in temperature decrease the hydration around the two parts, and the observed minimum on the cmc-temperature plot reflects the effect of rising temperature on a balance between a gradual dehydration of the hydrophobic part (this promotes micellization) and a partial dehydration of the hydrophilic part, leading to an increase in repulsion between polar headgroups. The partial dehydration can spread to the surface of 
micelles already formed. The variation of the equilibrium constant for the aggregation process with temperature was attributing only to the temperature coefficient of the $\mathrm{cmc}$. The standard Gibbs energy change per mole of monomer for these penicilins are plotted in Figs. (3, 4 and 5). In Fig. (3) only the energies for penicillins with one stage of aggregation are plotted meanwhile in Fig. (4) and Fig. (5) we have plotted the energies for penicillins corresponding to the first and second stage of aggregation respectively.

Table 1 shows the temperature dependence of the enthalpy and entropy of aggregation per mole for each self aggregation. Standard Gibbs energy are of the same order for all these penicillin, taking into account that for penicillins with two critical points, the total Gibbs energy will be the sum of both stages. It is interesting to observe the Gibbs energies for ampicillin, oxacillin and penicillin $G$. In the first stage ampicillin shows the greatest values, however oxacillin will be the greater in the second. If we rescue the aggregation numbers obtained from light scattering. In the first stage we obtain two monomers for penicillin $G$ and oxacillin meanwhile for ampicillin are three. In the second stage the number of aggregates that join themselves are two for ampicillin, three for penicillin $\mathrm{G}$ and four for oxacillin, indicating that Gibbs energy reach the higher values for the greater aggregation numbers. Positive values of standard enthalpy are generally attributed to the release of structured water from the hydration layers around the hydrophobic parts of the molecule during the formation of aggregates. Such hydrophobic interactions become increasingly insignificant with the partial breakdown of the structure of water as the temperature is increased and the aggregation becomes primarily an enthalpic process, the negative enthalpy values suggest the importance of the London-dispersion interaction as the major force for aggregation. For the second stage the slope of the enthalpy is lower for ampicillin and penicillin $G$, and thus in this case we have small aggregates which minimize the contact surface of monomers with water, the hydrophobic interactions being less important. However oxacillin exhibits the opposite behavior, this event it could be attrib-

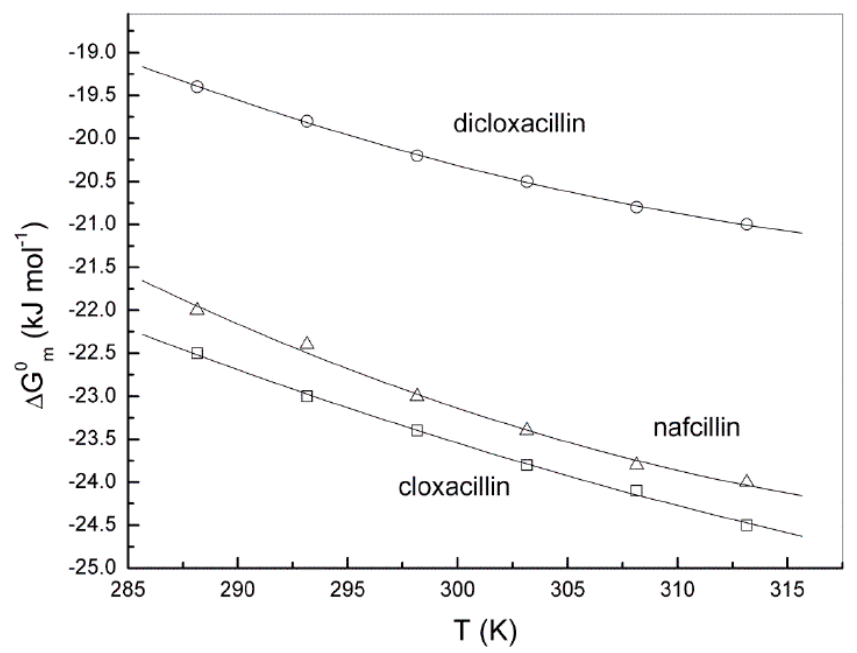

Fig. (3). Standard Giggs energy of aggregate formation per mol of monomer for: $(\square$ )cloxacillin; $(\bigcirc)$ dicloxacillin and $(\triangle)$ nafcillin. $\square \bigcirc \triangle$ uted to high aggregation number in the second stage (related to the first). Standard entropy calculated from Gibbs energy and enthalpy for these penicillins decrease progressively with temperature showing that at temperatures below the critical concentration minimum the aggregation is driven solely by the positive $\Delta S^{o}$.

Another interesting feature arise if we correlate the enthalpic and entropic contributions to micellization $\Delta H_{m}^{o}$ versus $\Delta S_{m}^{o}$. Thus, we obtain linear relationship, the so-called compensation phenomenon. Experimental data can be fitted to equation $\Delta H_{m}^{o}=\Delta H^{o *}{ }_{m}+T_{c} \Delta S_{m}^{o}$, where $T_{c}$ is the compensation temperature. Lumry and Rajender [78], Sugihara et al. [79] have interpreted this phenomenon as micelle formation being a desolvation process form a monomeric state to a micellar state and vice verse, i. e., in micelle formation the water molecules surrounding amphiphilic molecules play a more important role that the surfactant molecules themselves. When hydrates ionic groups of amphiphiles are bound with surrounding water molecules, the process leads to an energetic stabilization (negative enthalpy change). At the same time, the motion of water molecules bound to ionic groups causes a decrease in entropy. In the process of micelle formation (translation of hydrophobic groups from bulk to micelle), the entropy increase and a negative change in free energy takes place. The entalphy changes in conjunction with the entropy and switches its sign from negative to positive at a certain temperature, the compensation temperature. The interpretation most commonly accepted for $T_{c}$ is that it simply reflects the cooperation of enthalpy with entropy for the systems studied under determined conditions. We can then state this value as characteristic for the micellization process of the penicillins and betablockers in water in the temperature range of $288.15 \mathrm{~K}$ to $313.15 \mathrm{~K}$. The intercept correspond to the entropy change at a specific temperature, giving $\Delta H_{m}^{o}=0$. In this situation, the driving force of micelle formation comes only from the entropy term, i. e., the standard Gibbs energy of micellization only has an entropy term. The temperature at which this takes place is the minimum of the cmc-temperature plots. As it has been suggested,

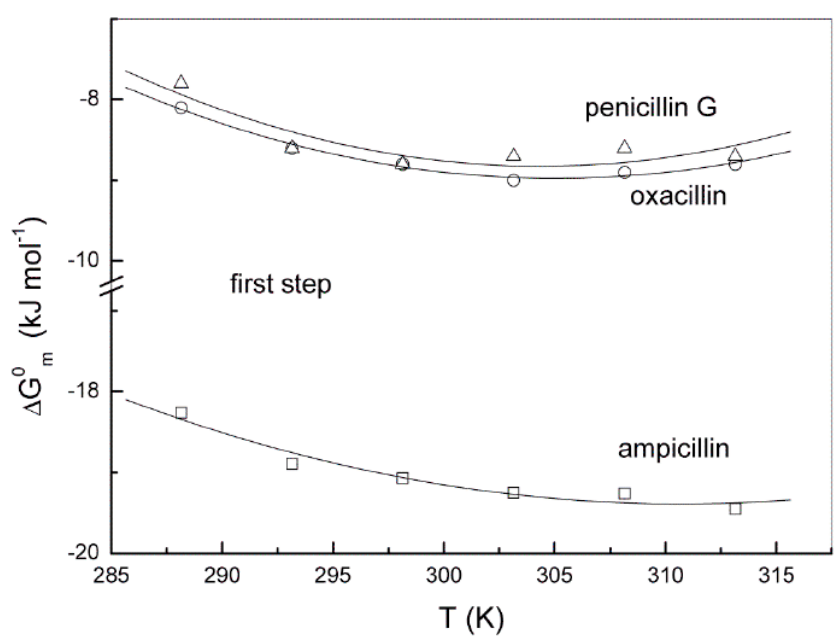

Fig. (4). Standard Giggs energy of first stage aggregate formation per mol of monomer for: $(\square)$ ampicillin; $(\bigcirc)$ oxacillin and $(\triangle)$ penicillin. 
it would be an index of the hydrophobic-hydrophilic balance[27e].

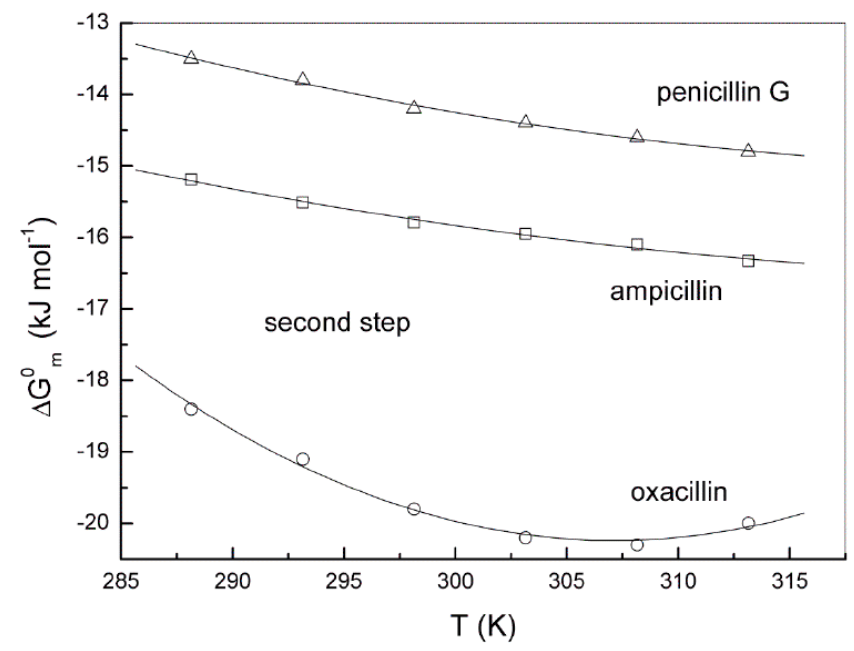

Fig. (5). Standard Gibbs energy of second stage aggregate formation per mol of monomer for: $(\square)$ ampicillin; $(\bigcirc)$ oxacillin and $(\triangle)$ penicillinG

\section{COMPLEXATION WITH PROTEINS}

Molecular self-assembly is central to molecular medicine, one of the most active trend in health area. Much of biotechnological, medical and drug delivery systems aims to predict their behavior on the basis of the set of molecules involved, mainly proteins and drugs. Understanding the interactions between these molecules is therefore crucial to such efforts [80]. Understanding protein-drug interactions is central to drug design and the discovery of new medicines to benefit human health. Also, recent advances in nanotechnology or hybrid nanomaterial have triggered the enthusiasm for these complexes [81]. The ability to identify small molecule ligands for any protein of interest has far-reaching implications, both for the elucidation of protein function and for the development of novel pharmaceuticals [82].

The interaction of a range of penicillins, nafcillin, cloxacillin, dicloxacillin, and flucloxacillin with human serum albumin (HSA) in aqueous solution at $\mathrm{pH} 7.4$ at $25^{\circ} \mathrm{C}$ [83] have been investigated by means of zeta potential measurements. The interest in the interactions of penicillins with HSA arises from reports of allergic reactions in some subjects to penicillin and other $\beta$-lactam antibiotics and to evidence of haptenation of penicillin by conjugation with serum proteins, resulting in the formation of $\operatorname{IgE}$ antibodies. The interaction of penicillins with HSA arises both from the hydrophobic nature of the drugs and the presence of hydrophobic cavities in the protein structure [84]. The $\xi$-potentials of HSA became more negative as the negatively charged penicillins bind to it. The variations of $\zeta$-potential with drug concentration have been interpreted in terms of a modification of the theory of Ottewill and Watanabe (OW) to take into

Table 1. Standard enthalpy ( $\Delta H_{m}^{0}$ in $\left.\mathrm{kJ} \mathrm{mol}^{-1}\right)$ and entropy $\left(\Delta S_{m}^{0}\right.$ in $\left.\mathbf{k J ~ m o l}^{-1} \mathrm{~K}^{-1}\right)$ of micelle formation per mol in water at different temperatures ( $\mathrm{T}$ in $\mathrm{K})$.

\begin{tabular}{|c|c|c|c|c|c|c|}
\hline \multirow[b]{2}{*}{$\mathbf{T}$} & \multicolumn{2}{|c|}{ Cloxacillin } & \multicolumn{2}{|c|}{ Dicloxacillin } & \multicolumn{2}{|c|}{ Nafcillin } \\
\hline & $\Delta H_{m}^{0}$ & $\Delta S_{m}^{0}$ & $\Delta H_{m}^{0}$ & $\Delta S_{m}^{0}$ & $\Delta H_{m}^{0}$ & $\Delta S_{m}^{0}$ \\
\hline 288.15 & 2.9 & 88 & 4.5 & 82 & 7.5 & 102 \\
\hline 293.15 & 1.2 & 82 & 2.2 & 75 & 3.7 & 89 \\
\hline 298.15 & -0.5 & 76 & -0.3 & 66 & -0.3 & 75 \\
\hline 303.15 & -2.4 & 70 & -2.9 & 58 & 4.7 & 61 \\
\hline 308.15 & -4.4 & 63 & -5.7 & 49 & -9.3 & 47 \\
\hline 313.15 & -6.5 & 57 & -8.8 & 40 & -14.2 & 31 \\
\hline
\end{tabular}

\begin{tabular}{|c|c|c|c|c|c|c|c|c|c|c|c|c|}
\hline \multirow[b]{3}{*}{$\mathbf{T}$} & \multicolumn{4}{|c|}{ Ampicillin } & \multicolumn{4}{|c|}{ Oxacillin } & \multicolumn{4}{|c|}{ Penicillin G } \\
\hline & \multicolumn{2}{|c|}{ First } & \multicolumn{2}{|c|}{ Second } & \multicolumn{2}{|c|}{ First } & \multicolumn{2}{|c|}{ Second } & \multicolumn{2}{|c|}{ First } & \multicolumn{2}{|c|}{ Second } \\
\hline & $\Delta H_{m}^{0}$ & $\Delta S_{m}^{0}$ & $\Delta H_{m}^{0}$ & $\Delta S_{m}^{0}$ & $\Delta H_{m}^{0}$ & $\Delta S_{m}^{0}$ & $\Delta H_{m}^{0}$ & $\Delta S_{m}^{0}$ & $\Delta H_{m}^{0}$ & $\Delta S_{m}^{0}$ & $\Delta H_{m}^{0}$ & $\Delta S_{m}^{0}$ \\
\hline 288.15 & 7.6 & 89.8 & 3.1 & 63 & 19.7 & 96 & 38.0 & 195 & 22.5 & 30 & 8.1 & 21 \\
\hline 293.15 & 3.4 & 76.1 & 1.3 & 57 & 12.0 & 70 & 23.9 & 146 & 13.6 & 22 & 5.5 & 19 \\
\hline 298.15 & -2.5 & 55.6 & -0.7 & 50 & 3.7 & 42 & 8.7 & 95 & 4.0 & 12 & 2.6 & 16 \\
\hline 303.15 & -8.8 & 34.4 & -2.8 & 43 & -5.1 & 12 & -7.5 & 42 & -6.3 & 2 & -0.5 & 14 \\
\hline 308.15 & -15.6 & 12.0 & -5.0 & 36 & -14.5 & -18 & -24.7 & -14 & -17.2 & -8 & -3.7 & 10 \\
\hline 313.15 & -22.7 & -10.4 & -7.3 & 28 & -24.6 & -50 & -43.2 & -74 & -28.9 & -20 & -7.2 & 7 \\
\hline
\end{tabular}


account the cooperativity of drug binding in terms of the Hill equation [85]. The Hill coefficients for the drugs were all greater than unity and diagnostic of positive cooperativity on binding [86]. The plots and the Gibbs energies per drug molecule bound as a function of the number of drug molecules bound per protein molecule are closer to the experimental values from equilibrium dialysis experiments than when the Langmuir isotherm is used. The modified OW theory was consistent with the clustering of drug molecules on the polypeptide chain of the protein and a description of the resulting complexes in which the protein is covered in micelle-like aggregates. In all studied systems the standard Gibbs energies of adsorption evaluated from the above equation have shown that Gibbs energies are large and negative at low values of drug concentration where binding to the high energy sites takes place, and become less negative as more drug molecules bind suggesting a saturation process [87]. As an example, calculated values of the number of adsorption sites and Gibbs energies of different drugs including: penicillins $[88,89]$, betablockers [27c] or antidepressants [90]'onto human serum albumin can be consulted in Table 2 .

Table 2. Number of adsorption sites, $N_{1}$, and standard gibbs energy of adsorption, $\Delta G_{a d s}^{0}$, of some drug onto human serum albumin.

\begin{tabular}{|c|c|c|}
\hline Drug & $\boldsymbol{N}_{\boldsymbol{I}}\left(\mathbf{m}^{-2}\right)$ & $\Delta G_{a d s}^{0}\left(\mathbf{k J ~ m o l}{ }^{-1}\right)$ \\
\hline \hline Cloxacillin & $8.34 \times 10^{15}$ & -7.7 \\
\hline Dicloxacillin & $2.37 \times 10^{16}$ & -10.2 \\
\hline Nafcillin & $1.09 \times 10^{16}$ & -7.7 \\
\hline Propranolol & $5.48 \times 10^{15}$ & -14.2 \\
\hline Verapamil & $4.09 \times 10^{10}$ & -7.8 \\
\hline Amitriptyline & $1.03 \times 10^{17}$ & -4.1 \\
\hline Nortriptyline & $9.1 \times 10^{14}$ & -7.9 \\
\hline Imipramine & $9.8 \times 10^{15}$ & -5.7 \\
\hline Desipramine & $6.5 \times 10^{15}$ & -5.8 \\
\hline
\end{tabular}

As an illustrative example, the adsorption of nafcillin on catalase at different $\mathrm{pHs}$ can be useful [91]. Obtained isotherms at $\mathrm{pH} 3.2$ reveals an initially binding of the anionic nafcillin to cationic residues, followed by hydrophobic binding at the drug concentration approaching the critical micellar concentration (cmc) of the drug. The isotherm passes through maxima. Such behavior has been attributed to the activity of long-chain ions going through a maximum in the vicinity of the cmc. At $\mathrm{pH} 7.4$ and 10.0, specific binding to cationic sites is lost, and only hydrophobic binding occurs. The number of drug molecules per amino acid residue was $0.37,5.4$, and 4.64 for $\mathrm{pH} 3.2,7.4$, and 10.0, respectively.

The complexation of nafcillin, cloxacillin, dicloxacillin, and flucloxacillin, with human serum albumin (HSA) in aqueous solution, $\mathrm{pH} 7.4$, has been also analyzed by equilibrium dialysis [92]. In this case, the binding process is largely non- specific. The binding isotherms for the drugs range up to values of 3000 for nafcillin and cloxacillin, 1200 for di- cloxacillin, and 2000 for flucloxacillin. Human serum albumin consists of 585 amino acids 18 so binding of 3000 drug molecules corresponds to approximately 5 drug molecules per amino acid residue; this suggests clustering of drug molecules along the polypeptide chain. Such a cluster size corresponds closely to the aggregation numbers of the drug micelles, which at an ionic strength of $0.1 \mathrm{M}$ were found to be 5 (cloxacillin), 5 (dicloxacillin), and 4 (flucloxacillin). However, these data do not allow to draw any conclusions about the uniformity of binding along the polypeptide chain, although it is likely that binding is greater (and cluster size larger) around hydrophobic aminoacid residues and in hydrophobic cavities in the protein and less around hydrophilic residues [93]. On the other hand, binding strength as represented by the Gibbs energies of binding increases (becomes more negative) in the sequence dicloxacillin $>$ nafcillin $>$ flucloxacillin $>$ cloxacillin, although the differences between the latter three are not large. The larger Gibbs energy of binding for the dichloro drug are related to a contribution from dipole interactions that may be greater than in the case of the monohalide drugs, cloxacillin and flucloxacillin. The nafcillin interaction is stronger than the monohalide interactions because of a greater hydrophobic contribution arising from the naphthalene ring.

UV difference spectroscopy have been utilized to study the interaction of the amphiphilic drug propranolol hydrochloride with human haemoglobin $(\mathrm{HH})$ and human serum albumin (HSA) in aqueous solution at different pHs [27c, 94]. Addition of propranolol results in the development of an absorption band in the region of $410 \mathrm{~nm}$ that is positive with respect to native $\mathrm{HH}$, arising from the extended $\pi$-electron system of the porphyrin ring. For the HSA/propranolol systems the main peaks were observed at 410, 413, and $417 \mathrm{~nm}$ for pHs 2.5, 4.9, and 8.0, respectively. The UV absorption of human serum albumin (HSA) increased smoothly with increase of concentration of added propranolol hydrochloride at all pHs as a consequence of adsorption of the drug ions. In contrast, an abrupt change in these properties was observed for the human haemoglobin $(\mathrm{HH})$ /propranolol system at a concentration of approximately $0.05 \mathrm{~mol} \mathrm{kg-1}$ at $\mathrm{pH} 2.5$. This change has been attributed to a tetramer to dimer transition arising from the increase of concentration of drug which functions as an electrolyte.

Fig. (6) shows static light scattering data for HSA in aqueous solutions containing between 0.015 and $0.15 \mathrm{molkg}$ 1 nafcillin [89]. In this treatment of the data nafcillin has been considered as an electrolyte, so the system is acting as a binary system formed by the protein complex as solute, and the nafcillin water as solvent. Molar masses, derived from the intercepts of Fig. (6) increased linearly with concentration of added drug. The molar mass obtained using the Debye equation for the HSA in pure water was $7.9 \times 10^{4} \mathrm{~g} \mathrm{~mol}^{-1}$, which corresponds to an association number $N=M_{w} / M_{0}$ (where $M_{0}$ is the molecular weight of the HSA, $6.641 \times 10^{4} \mathrm{~g}$ $\mathrm{mol}^{-1}$ ) of 1.19 indicating only limited association. The average number of nafcillin molecules adsorbed onto the protein can be obtained from the difference between the molar mass of HSA $\left(6.6 \times 10^{4} \mathrm{~g} \mathrm{~mol}^{-1}\right)$ and that of the complex, divided by the molar mass of nafcillin $\left(454.5 \mathrm{~g} \mathrm{~mol}^{-1}\right)$. The average number of nafcillin molecules adsorbed onto HSA derived by this method changes with nafcillin concentration from 
171 (at $0.015 \mathrm{~mol} \mathrm{~kg}^{-1}$ ) to 1075 (at $0.15 \mathrm{~mol} \mathrm{~kg}^{-1}$ ). Based on these results it can be deduced that at small drug concentrations, individual drug molecules bind to the protein which results in small changes in protein structure. On the other hand, drug molecules at higher concentrations aggregate to form micelle-like clusters along the unfolded polypeptide chains of the protein. HSA has exhibit the same pattern in the presence of other drugs such as cloxacillin, dicloxacillin [95] and verapamil [90]. This behavior has been studied not only by experimental techniques but also by a theoretical formalism based on the combination of the BrunauerEmmet-Teller multilayer adsorption model with an electrolytic adsorbate. The experimental binding processes are correctly predicted by this noncooperative binding model and it is demonstrated to be due to the ionic character of the drug. The effect of the hydrophobic interactions between drug monomers is also a matter of study, and it is proven that this number increases with increasing hydrophobic character of the drug molecule [96].

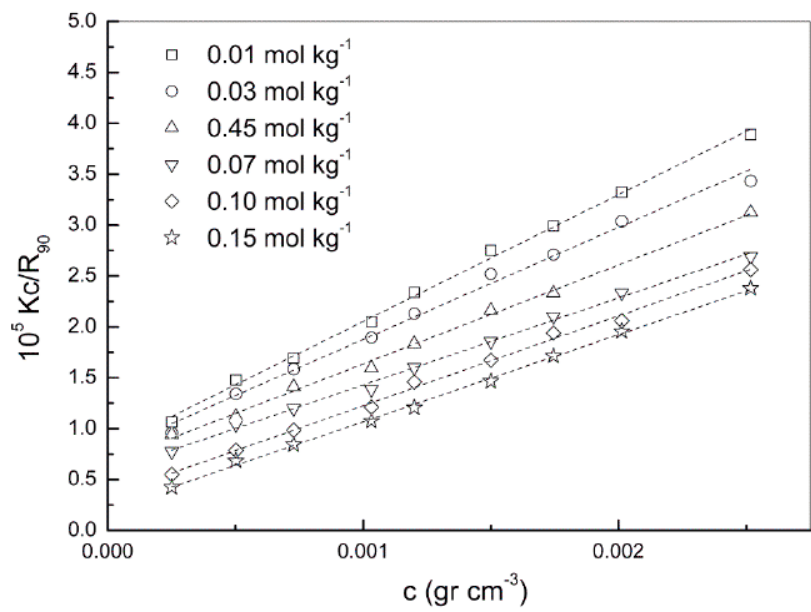

Fig. (6). $\mathrm{Kc} / \mathrm{R}_{90}$, as a function of the human serum albumin concentration in aqueous solutions of the drug nafcillin.

The interaction potential between the protein/drug complexes and their stability can be determined from the dependence of diffusion coefficients on protein concentration. For this purpose, the DLVO theory can be refined or modified by including other terms in the potential $V(x)$, the electrostatic potential can be evaluated more accurately by using the nonlinear Poisson- Boltzmann equation or by other models. Finally, the physical meaning of the cutoff distance can be defined on the basis of ionic strength dependence. Fig. (7) shows the $V(x) / k_{\mathrm{B}} T$ curves for the system HSA/propranolol, for a set of propranolol concentrations, showing the predominance of electrostatic repulsion at each concentration, which leads to a very stable dispersion. Increased adsorption with increasing propranolol concentration leads to screening of the electrostatic potential and the increasing importance of London-van der Waals attraction. Other typical values of $A$ and $q$ obtained for HSA in the presence of different drugs were: $A=0.41 \times 10^{-22} \mathrm{~J}$ and $q=2.2 \mathrm{uec}$ (unit of electron charge) in the presence of propranolol [27c]; $A=4.11 \times 10^{-23}$ $\mathrm{J}$ and $q=0.35 \mathrm{uec}$ in the presence of nafcillin [89]; $A=$ $4.1 \times 10^{-23} \mathrm{~J}$ and $q=1.13 \mathrm{uec}$ in the presence of cloxacillin; $A$ $=2.5 \times 10^{-23} \mathrm{~J}$ and $q=1.01 \mathrm{uec}$ in the presence of dicloxacillin $[88]$.

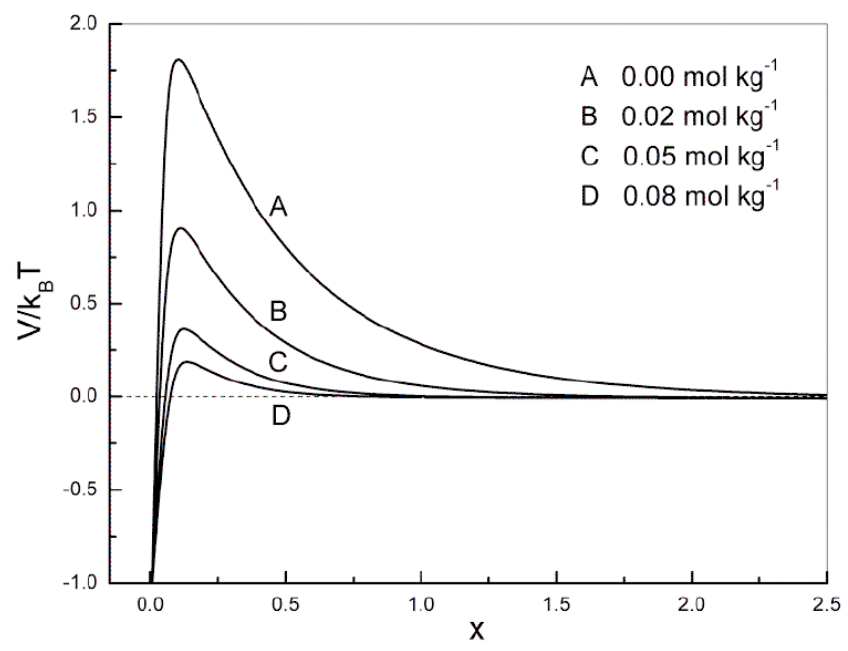

Fig. (7). Plots of the pair interaction potential $(V)$, between HSA/propranolol complexes, as a function of the reduced and normalized distance $(\mathrm{x})$ at the propranolol concentrations indicated in the graph. HSA concentration $0.125 \% \mathrm{w} / \mathrm{v}$.

Different thermodynamic and statistical mechanical approaches to describing unfolding, stability and energy of proteins linked to a ligand have been discussed in literature [97]. Early studies have demonstrated the usefulness of Differential Scanning microcalorimetry (DSC) in understanding the drug-resistance mechanism caused by mutations. When drugs bind to a protein, the energy of ligand binding adds incrementally to the protein's stabilization energy and the protein's $T_{\mathrm{m}}$ is increased by an amount that is proportional to the ligand binding energy. Thermodynamic analysis of the $\Delta T_{\mathrm{m}}$, or thermal shift, associated with ligand binding can be semiquantitatively related to ligand binding affinity, subject to reasonable assumptions regarding the ligand binding enthalpies of drug-like compounds [98]. Enteric-coated bovine serum albumin (BSA) nanospheres carrying cyclodextrin complex were performed for indomethacin delivery. The inclusion complex and the nanospheres were characterized by DSC analysis. The thermal behavior of albumin nanospheres showed no phenomena in the temperature interval. Albumin nanospheres carrying inclusion complex showed disappearance of the endothermal peak of indomethacin indicating the the strong drug interaction with albumin or albumin/betacyclodextrin carrier. The results showed that indomethacin solubility can be increased by complexation with protein/drug interaction with albumin nanospheres [99]. DSC studies on amorphous trehalosewater systems with embedded proteins (myoglobin, lysozyme, BSA, hemoglobin) were performed. From DSC upscans the thermodynamic properties of the matrix (glass transition) and the functional properties of the encapsulated protein (thermal denaturation) were calculated. At high hydration, the presence of the proteins increases the glass transition temperature of the encapsulating matrix. An increase of the protein thermal stability is observed in the whole hydration range, a finding potentially of high biotechnological relevance [100].

DSC thermograms have allowed characterizing the processes involved in synthesis of the antibody-drug conjugate Trastuzumab-DM1 (a semysinthetic ansamaclolide) [101]. 
The thermal denaturation of ovalbumin, lysozyme, myoglobin and fibrinogen at different 3-(2-Benzothiazolylthio)propanesulfonic acid (BTS) concentrations have been investigated using DSC. The thermal denaturation of the four proteins was completely irreversible. A major effect is observed in the case of myoglobin, the protein with the highest $\alpha$ helical secondary structure $(75 \%)$. This could be related with the fact that backbone hydrogen bonds of $\alpha$-helix are generally slightly weaker than those found in $\beta$-sheets. Thus, they are readily attacked by the surrounding BTS molecules [102].

The complex formed due to the interaction of the amphiphilic betablocker acebutolol with fibrinogen in a buffer solution $(50 \mathrm{mN}$ glycine, $\mathrm{pH}$ of 8.5$)$ was investigated. The transitions from the native to the unfolded state of fibrinogen induced by acebutolol have been found to be a multiple step process with intermediate molten globule states [103].

Isothermal Titration Calorimetry [104] experiments show that the interaction of cytochrome $c$ with ferulic acid is driven by a moderately favorable entropy increase in combination with a less favorable enthalpy decrease for the first binding site of the protein [105]. The interaction energies of the NimA protein and the antibiotics: metronidazole, tinidazole, ornidazole and dimetrazoleantibiotic were are studied by ITC. Measurements found that one NimA dimer has two antibiotic binding sites which were not affected by mutation. The enthalpy release upon binding to NimA for the four drugs studied was relatively low, because of the drug binding is mainly entropy driven and along with the hydrophobic drug binding site [106]. Evaluation of binding of vismodebig (a selective Hedgehog pathway inhibitor) with human and rat AAGs and albumins have confirmed that binding is rapidly and completely reversible. The data suggest that as compound enters the bloodstream, it will preferentially bind to available binding sites on AAG rather than albumin [107]. ITC studies further demonstrate that quinacrine can serve as a targeting ligand for specific delivery of additional therapeutic molecules or imaging agents to the receptoroverexpressing cancer cells implicated in breast and prostate cancers. Pertinent to this targeting utility, it would be possible to apply the concept of multivalent ligand design, in which even suboptimal targeting capability can be enhanced through multivalent tight binding [108].

\section{SUMMARY AND OUTLOOKS}

In summary, we have highlighted the advances in drug self-assemblies and discuss in detail their synthesis, physical properties and applications as well as their interaction with proteins. We also examine, evaluate and link various bioinformatics procedures applied to drug self-assembly and it interaction with biological units. Bio-informatic strategies are new tools to link the biological networks with specific units relevant to clinical disease, facilitating the integration of experimental data with sophisticated statistical approaches. The examples discussed clearly underline the significant advances made on the compression of drug selfassembly and it application in medicinal chemistry. Even though the vast effort made on the bio-inspired supramolecular self-assembly topic, we believe that more work needs to be dedicated to incorporate the information related to the drugs colloidal aggregation in the following areas: (i) smart biomaterials development; (ii) synthesis of naturally mimicking hierarchical self-assemblies structures; (iii) incorporation of bio-functional segments to improve the biofunctionality and bioactivity of bio- medical devices.

\section{CONFLICT OF INTEREST}

The author(s) confirm that this article content has no conflicts of interest.

\section{ACKNOWLEDGEMENTS}

The authors acknowledge partial financial support from Xunta de Galicia (Project No. 10PXIB206258PR), Universidad Nacional del Sur (PGI 24/ZQ07), Concejo Nacional de Investigaciones Científicas y Técnicas de la República Argentina (CONICET, PIP-11220100100072).

\section{REFERENCES}

[1] Whitesides, G. M.; Grzybowski, B., Self-Assembly at All Scales. Science, 2002, 295 (5564), 2418-2421.

[2] (a) Marini, D. M.; Hwang, W.; Lauffenburger, D. A.; Zhang, S.; Kamm, R. D., Left-Handed Helical Ribbon Intermediates in the Self-Assembly of a $\beta$-Sheet Peptide. Nano Letters, 2002, 2 (4), 295-299; (b) Hassan, N.; Ruso, J. M.; Piñeiro, A. n., Hydrogenated/Fluorinated Catanionic Surfactants as Potential Templates for Nanostructure Design. Langmuir, 2011, 27 (16), 9719-9728.

[3] Nakano, T.-Y.; Sugihara, G.; Nakashima, T.; Yu, S.-C., Thermodynamic Study of Mixed Hydrocarbon/Fluorocarbon Surfactant System by Conductometric and Fluorimetric Techniques. Langmuir, 2002, 18 (23), 8777-8785.

[4] (a) Nagai, A.; Nagai, Y.; Qu, H.; Zhang, S., Dynamic Behaviors of Lipid-Like Self-Assembling Peptide A6D and A6K Nanotubes. Journal of Nanoscience and Nanotechnology, 2007, 7 (7), 22462252; (b) Blanco, E.; Piñeiro, A. n.; Miller, R.; Ruso, J. M.; Prieto, G.; Sarmiento, F. 1., Langmuir Monolayers of a Hydrogenated/Fluorinated Catanionic Surfactant: From the Macroscopic to the Nanoscopic Size Scale. Langmuir, 2009, 25 (14), 8075-8082.

[5] Blanco, E.; Verdes, P. V.; Ruso, J. M.; Prieto, G.; Sarmiento, F., Interactions in binary mixed systems involving betablockers with different lipophilicity as a function of temperature and mixed ratios. Colloids and Surfaces A: Physicochemical and Engineering Aspects, 2009, 334 (1-3), 116-123.

[6] Krishnan, R. S. G.; Thennarasu, S.; Mandal, A. B., Self-assembling characteristics of 5 -( $\alpha$-acetamido- $\alpha$-benzyl $)$ methyl-5'-methyl imidazolidine-2,4-dione - A hydantoin drug. Chemical Physics, 2003, 291 (2), 195-205.

[7] Hauser, E. A.; Marlowe, G. J., The Colloidal Phenomena of Antibiotics. The Journal of Physical and Colloid Chemistry, 1950, 54 (8), 1077-1087.

[8] Kumler, W. D.; Alpen, E. L., Surface Tension and Conductivity of Penicillin Salts. Science, 1948, 107 (2787), 567.

[9] McBain, J. W.; Huff, H.; Brady, A. P., Penicillin G as Electrolyte and Colloidal Electrolyte. J. Am. Chem. Soc., 1949, 71 (1), 373374.

[10] Hocking, C. S., Light-scattering Studies on Penicillin G Solutions Nature, 1951, 168 (4271), 423-424.

[11] Few, A. V.; Schulman, J. H., On the surface chemistry of sodium penicillin G. Biochimica et Biophysica Acta, 1953, 10 (0), 302-310.

[12] Ong, J. T. H.; Kostenbauder, H. B., Effect of self-association on rate of penicillin $\mathrm{G}$ degradation in concentrated aqueous solutions. Journal of Pharmaceutical Sciences, 1975, 64 (8), 1378-1380.

[13] Funasaki, N.; Hada, S.; Neya, S., Self-Association of Penicillins in Aqueous Solution as Revealed by Gel Filtration Chromatography. CHEMICAL \& PHARMACEUTICAL BULLETIN, 1994, 42 (4), 779-785.

[14] Thakkar, A. L.; Wilham, W. L., Self-association of benzylpenicillin in aqueous solution: $1 \mathrm{H}$ nuclear magnetic resonance study. Journal 
of the Chemical Society D: Chemical Communications, 1971, 0 (7), 320-322.

[15] Attwood, D.; Agarwal, S. P., Light scattering studies on micelle formation by some penicillins in aqueous solution. Journal of Pharmacy and Pharmacology, 1984, 36 (8), 563-564.

[16] (a) Messina, P.; Pieroni, O.; Vuano, B.; Ruso, J.; Prieto, G.; Sarmiento, F., Thermodynamic study of functionalized calix[n]arene and resorcinol[n] arene monolayers spreaded at an aqueous pendant drop. $J$ Incl Phenom Macrocycl Chem, 2010, 67 (3-4), 343-352; (b) Messina, P.; Ruso, J.; Prieto, G.; FernándezLeyes, M.; Schulz, P.; Sarmiento, F., Ca2+- and Mg2+-induced molecular interactions in a dehydrocholic acid/didodecyldimethylammonium bromide mixed monolayer. Colloid Polym Sci, 2010, 288 (4), 449-459.

[17] Hassan, N.; Ruso, J. M.; Gonzalez-Perez, A., Self-assembling drugs: A new therapeutic strategy. Soft Matter, 2011, 7 (11), 51945199 .

[18] (a) Drummond, C. J.; Fong, C., Surfactant self-assembly objects as novel drug delivery vehicles. Current Opinion in Colloid \& Interface Science, 1999, 4 (6), 449-456; (b) Schreier, S.; Malheiros, S. V. P.; de Paula, E., Surface active drugs: self-association and interaction with membranes and surfactants. Physicochemical and biological aspects. Biochimica et Biophysica Acta (BBA) Biomembranes, 2000, 1508 (1-2), 210-234; (c) Rösler, A.; Vandermeulen, G. W. M.; Klok, H.-A., Advanced drug delivery devices via self-assembly of amphiphilic block copolymers. Advanced Drug Delivery Reviews, 2012, 64, Supplement (0), 270279.

[19] Ruso, J. M.; Attwood, D.; Taboada, P.; Suárez, M. J.; Sarmiento, F.; Mosquera, V., Activity and Osmotic Coefficients of Promethazine and Chlorpromazine Hydrochlorides in Aqueous Solutions of Low Ionic Strength. Journal of Chemical \& Engineering Data, 1999, 44 (5), 941-943.

[20] Attwood, D.; Blundell, R.; Mosquera, V.; Garcia, M., Association and Surface Properties of Amphiphilic Benzodiazepine and Benzothiazepine Drugs in Aqueous Solution. Journal of Colloid and Interface Science, 1993, 161 (1), 19-23.

[21] Attwood, D.; Tolley, J. A., Self-association of analgesics in aqueous solution: association models for codeine, oxycodone, ethylmorphine and pethidine. Journal of Pharmacy and Pharmacology, 1980, 32 (1), 761-765.

[22] Epand, R. M.; Vogel, H. J., Diversity of antimicrobial peptides and their mechanisms of action. Biochimica et Biophysica Acta (BBA) Biomembranes, 1999, 1462 (1-2), 11-28.

[23] (a) Besada, L.; Martinez-Landeira, P.; Seoane, L.; Prieto, G.; Sarmiento, F.; Ruso, J. M., A study of the behaviour of ampicillin in aqueous solution and thermodynamic characterization of its aggregation. Molecular Physics, 2001, 99 (24), 2003-2009; (b) Varela, L. M.; Rega, C.; Suarez-Filloy, M. J.; Ruso, J. M.; Prieto, G.; Attwood, D.; Sarmiento, F.; Mosquera, V., Self-Association of Penicillin V in Aqueous Solution. Langmuir, 1999, 15 (19), 62856290.

[24] Taboada, P.; Ruso, J. M.; García, M.; Mosquera, V., Comparison of the thermodynamic properties of structurally related amphiphilic antidepressants in aqueous solution. Colloid Polym Sci, 2001, 279 (7), 716-720.

[25] Attwood, D.; Udeala, O. K., Aggregation of antihistamines in aqueous solution. Self-association of some pyridine derivatives. The Journal of Physical Chemistry, 1975, 79 (9), 889-892.

[26] Yokoyama, S.; Fujino, Y.; Kawamoto, Y.; Kaneko, A.; Fujie, T., Micellization of an Aqueous Solution of Piperidolate Hydrochloride in the Presence of Acetylcholine Chloride. CHEMICAL \& PHARMACEUTICAL BULLETIN, 1994, 42 (6), 1351-1353.

[27] (a) Ruso, J. M.; Attwood, D.; Rey, C.; Taboada, P.; Mosquera, V.; Sarmiento, F., Light Scattering and NMR Studies of the SelfAssociation of the Amphiphilic Molecule Propranolol Hydrochloride in Aqueous Electrolyte Solutions. The Journal of Physical Chemistry B, 1999, 103 (34), 7092-7096; (b) Mosquera, V.; amp; x; ctor; Ruso, J. M.; Attwood, D.; Jones, M. N.; Prieto, G.; Sarmiento, F., Thermodynamics of Micellization of Surfactants of Low Aggregation Number: The Aggregation of Propranolol Hydrochloride. Journal of Colloid and Interface Science, 1999, 210 (1), 97-102; (c) Ruso, J. M.; Attwood, D.; García, M.; Prieto, G.; Sarmiento, F.; Taboada, P.; Varela, L. M.; Mosquera, V., Interaction of Amphiphilic Propranolol Hydrochloride with
Haemoglobin and Albumin in Aqueous Solution. Langmuir, 2000, 16 (26), 10449-10455; (d) Ruso, J. M.; Taboada, P.; Attwood, D.; Mosquera, V.; Sarmiento, F., Determination of the aggregation properties of weakly self-associating systems by NMR techniques: the self-association of propranolol hydrochloride in aqueous electrolyte solution. Physical Chemistry Chemical Physics, 2000, 2 (6), 1261-1265; (e) Ruso, J. M.; González-Pérez, A.; Prieto, G.; Sarmiento, F., Thermodynamic Study of Self-Assembly Behavior of Propranolol Hydrochloride in Aqueous Solutions as a Function of Electrolyte Concentration and Temperature. Journal of Chemical \& Engineering Data, 2003, 48 (6), 1597-1602; (f) Ruso, J. M.; Lopez-Fontan, J. L.; Prieto, G.; Sarmiento, F., The self-association of acebutolol: Conductometry and light scattering. The Journal of Chemical Physics, 2003, 118 (13), 5964-5970; (g) Ruso, J. M.; González-Pérez, A.; Prieto, G.; Sarmiento, F., A volumetric study of two related amphiphilic beta-blockers as a function of temperature and electrolyte concentration. Colloids and Surfaces B: Biointerfaces, 2004, 33 (3-4), 165-175.

[28] Kitagawa, N.; Oda, M.; Totoki, T., Possible Mechanism of Irreversible Nerve Injury Caused by Local Anesthetics: Detergent Properties of Local Anesthetics and Membrane Disruption. Anesthesiology, 2004, 100 (4), 962-967.

[29] Fini, A.; Fazio, G.; Feroci, G., Solubility and solubilization properties of non-steroidal anti-inflammatory drugs. International Journal of Pharmaceutics, 1995, 126 (1-2), 95-102.

[30] Hassan, N.; Gárate, M. P.; Sandoval, T.; Espinoza, L.; Piñeiro, A. n.; Ruso, J. M., On the Self-Assembly of a Highly Selective Benzothiazole-Based TIM Inhibitor in Aqueous Solution. Langmuir, 2010, 26 (22), 16681-16689.

[31] Alam, M. S.; Ghosh, G.; Kabir ud, D., Light Scattering Studies of Amphiphilic Drugs Promethazine Hydrochloride and Imipramine Hydrochloride in Aqueous Electrolyte Solutions. The Journal of Physical Chemistry B, 2008, 112 (41), 12962-12967.

[32] Meller, J.; Elber, R., Computer Simulations of Carbon Monoxide Photodissociation in Myoglobin: Structural Interpretation of the B States. Biophysical journal, 1998, 74 (2), 789-802.

[33] Alder, B. J.; Wainwright, T. E., Studies in Molecular Dynamics. I. General Method. The Journal of Chemical Physics, 1959, 31 (2), 459-466.

[34] Rahman, A., Correlations in the Motion of Atoms in Liquid Argon. Physical Review, 1964, 136 (2A), A405-A411.

[35] McCammon, J. A.; Gelin, B. R.; Karplus, M., Dynamics of folded proteins. Nature, 1977, 267 (5612), 585-590.

[36] Levitt, M.; Warshel, A., Computer simulation of protein folding. Nature, 1975, 253 (5494), 694-698.

[37] Case, D. A.; Karplus, M., Dynamics of ligand binding to heme proteins. Journal of Molecular Biology, 1979, 132 (3), 343-368.

[38] Kuntz, I. D.; Blaney, J. M.; Oatley, S. J.; Langridge, R.; Ferrin, T. E., A geometric approach to macromolecule-ligand interactions. Journal of Molecular Biology, 1982, 161 (2), 269-288.

[39] Sundaram, K.; Mahajan, S., Theoretical studies on tricyclic antidepressants 3. Anaalysis of stereospecificity. Physiological Chemistry and Physics, 1981, 13 (5), 387-406.

[40] Jonsson, B.; Edholm, O.; Teleman, O., Molecular dynamics simulations of a sodium octanoate micelle in aqueous solution. The Journal of Chemical Physics, 1986, 85 (4), 2259-2271.

[41] Levitt, M.; Sharon, R., Accurate simulation of protein dynamics in solution. Proceedings of the National Academy of Sciences, 1988, 85 (20), 7557-7561.

[42] Swaminathan, S.; Beveridge, D. L.; Berman, H. M., Molecular dynamics simulation of a deoxydinucleoside-drug intercalation complex: dCpG/proflavin. The Journal of Physical Chemistry, 1990, 94 (11), 4660-4665.

[43] McDonald, J. J.; Brooks, C. L., Theoretical approach to drig design 2. Relative thermodynamics of inhibitor binding by chicken dihydrofolate-reductase to ethyl derivatives of trimethoprim substituted at 3'-position, 4'-position, and 5'-position. J. Am. Chem. Soc., 1991, 113 (6), 2295-2301.

[44] Heimstad, E.; Edvardsen, O.; Ferrin, T. E.; Dahl, S. G., Molecular structure and dynamics of tricyclic antidepressant drugs. European neuropsychopharmacology : the journal of the European College of Neuropsychopharmacology, 1991, 1 (2), 127-37.

[45] Kim, Y. H.; Gihm, S. H.; Park, C. R.; Lee, K. Y.; Kim, T. W.; Kwon, I. C.; Chung, H.; Jeong, S. Y., Structural characteristics of size-controlled self-aggregates of deoxycholic acid-modified 
chitosan and their application as a DNA delivery carrier. Bioconjugate Chem., 2001, 12 (6), 932-938.

[46] Marrink, S. J.; Mark, A. E., The Mechanism of Vesicle Fusion as Revealed by Molecular Dynamics Simulations. J. Am. Chem. Soc., 2003, 125 (37), 11144-11145.

[47] Selassie, C. D.; Mekapati, S. B.; Verma, R. P., QSAR: then and now. Curr Top Med Chem, 2002, 2 (12), 1357-1379.

[48] Dudek, A. Z.; Arodz, T.; Galvez, J., Computational methods in developing quantitative structure-activity relationships (QSAR): a review. Comb Chem High Throughput Screen, 2006, 9 (3), 213-28.

[49] Huibers, P. D. T.; Lobanov, V. S.; Katritzky, A. R.; Shah, D. O.; Karelson, M., Prediction of Critical Micelle Concentration Using a Quantitative Structure-Property Relationship Approach. 1. Nonionic Surfactants. Langmuir, 1996, 12 (6), 1462-1470.

[50] Devinsky, F.; Zamocka, J.; Lacko, I.; Polakovicova, M., QSAR and CAMM study of amphiphilic antimicrobially active 2,2'-bipyridyl monoammonium salts. Pharmazie, 1996, 51 (10), 727-731.

[51] Jalali-Heravi, M.; Konouz, E., Use of Quantitative Structure Activity Relationships in Prediction of CMC of Nonionic Surfactants. Quantitative Structure-Activity Relationships, 2000, 19 (2), 135-141.

[52] Anoune, N.; Nouiri, M.; Berrah, Y.; Gauvrit, J.-Y.; Lanteri, P., Critical micelle concentrations of different classes of surfactants: A quantitative structure property relationship study. J Surfact Deterg, 2002, 5 (1), 45-53.

[53] Katritzky, A. R.; Pacureanu, L.; Dobchev, D.; Karelson, M., QSPR Study of Critical Micelle Concentration of Anionic Surfactants Using Computational Molecular Descriptors $\dagger$. Journal of Chemical Information and Modeling, 2007, 47 (3), 782-793.

[54] Langham, A. A.; Khandelia, H.; Schuster, B.; Waring, A. J.; Lehrer, R. I.; Kaznessis, Y. N., Correlation between simulated physicochemical properties and hemolycity of protegrin-like antimicrobial peptides: Predicting experimental toxicity. Peptides, 2008, 29 (7), 1085-1093.

[55] Katritzky, A. R.; Pacureanu, L. M.; Slavov, S. H.; Dobchev, D. A.; Shah, D. O.; Karelson, M., QSPR study of the first and second critical micelle concentrations of cationic surfactants. Computers \& Chemical Engineering, 2009, 33 (1), 321-332.

[56] González-Díaz, H.; Arrasate, S.; Sotomayor, N.; Lete, E.; Munteanu, C. R.; Pazos, A.; Besada-Porto, L.; Ruso, J. M., MIANN Models in Medicinal, Physical and Organic Chemistry. Curr Top Med Chem, 2013, 13 (5), 619-641.

[57] (a) Gonzalez-Diaz, H.; Prado-Prado, F.; Ubeira, F. M., Predicting antimicrobial drugs and targets with the MARCH-INSIDE approach. Curr Top Med Chem, 2008, 8 (18), 1676-90; (b) González-Díaz, H.; González-Díaz, Y.; Santana, L.; Ubeira, F. M.; Uriarte, E., Proteomics, networks and connectivity indices. Proteomics, 2008, 8, 750-778; (c) González-Díaz, H.; Vilar, S.; Santana, L.; Uriarte, E., Medicinal Chemistry and Bioinformatics Current Trends in Drugs Discovery with Networks Topological Indices. Curr Top Med Chem, 2007, 7 (10), 1025-39; (d) GonzalezDiaz, H.; Arrasate, S.; Gomez-SanJuan, A.; Sotomayor, N.; Lete, E.; Besada-Porto, L.; Ruso, J. M., General Theory for Multiple Input-Output Perturbations in Complex Molecular Systems. 1. Linear QSPR Electronegativity Models in Physical, Organic, and Medicinal Chemistry. Curr Top Med Chem, 2013, 13 (14), 17131741 .

[58] (a) Santana, L.; Gonzalez-Diaz, H.; Quezada, E.; Uriarte, E.; Yanez, M.; Vina, D.; Orallo, F., Quantitative structure-activity relationship and complex network approach to monoamine oxidase a and B inhibitors. Journal of Medicinal Chemistry, 2008, 51 (21), 6740-51; (b) Santana, L.; Uriarte, E.; González-Díaz, H.; Zagotto, G.; Soto-Otero, R.; Mendez-Alvarez, E., A QSAR model for in silico screening of MAO-A inhibitors. Prediction, synthesis, and biological assay of novel coumarins. Journal of Medicinal Chemistry, 2006, 49 (3), 1149-56.

[59] Chandler, D., Interfaces and the driving force of hydrophobic assembly. Nature, 2005, 437 (7059), 640-647.

[60] Murphy, K. P., Hydration and convergence temperatures: on the use and interpretation of correlation plots. Biophysical Chemistry, 1994, 51 (2-3), 311-326.

[61] Tanford, C., Theory of micelle formation in aqueous solutions. The Journal of Physical Chemistry, 1974, 78 (24), 2469-2479.

[62] Young, T.; Abel, R.; Kim, B.; Berne, B. J.; Friesner, R. A., Motifs for molecular recognition exploiting hydrophobic enclosure in protein-ligand binding. Proceedings of the National Academy of Sciences, 2007, 104 (3), 808-813.

[63] (a) Ruso, J. M.; Attwood, D.; Taboada, P.; Mosquera, V.; Sarmiento, F., Light Scattering and NMR Studies on the SelfAggregation of Sodium n-Hexyl Sulfate in Aqueous Electrolyte Solution. Langmuir, 1999, 16 (4), 1620-1625; (b) Ruso, J. M.; Taboada, P.; Mosquera, V.; Sarmiento, F., Thermodynamics of Micellization of n-Alkyl Sulfates in an Alkaline Medium at Different Temperatures. Journal of Colloid and Interface Science, 1999, 214 (2), 292-296; (c) Blanco, E.; Messina†, P.; Ruso, J. M.; Prieto, G.; Sarmiento, F., Counterion effect on the solution and thermodynamic properties of lithium perfluoroalkanoates. Molecular Physics, 2005, 103 (24), 3271-3281; (d) Ruso, J. M.; Besada, L.; Martínez-Landeira, P.; Seoane, L.; Prieto, G.; Sarmiento, F., Interactions Between Liposomes and Cations in Aqueous Solution. Journal of Liposome Research, 2003, 13 (2), 131-145.

[64] Klevens, H. B., Structure and aggregation in dilate solution of surface active agents. J Am Oil Chem Soc, 1953, 30 (2), 74-80.

[65] Blanco, E.; Ruso, J. M.; Prieto, G.; Sarmiento, F., Partial molar volumes and partial molar adiabatic compressibilities of a short chain perfluorosurfactant: Sodium heptafluorobutyrate in aqueous solutions at different temperatures. The Journal of Chemical Thermodynamics, 2005, 37 (12), 1351-1355.

[66] Blanco, E.; Rodriguez-Abreu, C.; Schulz, P.; Ruso, J. M., Effect of alkyl chain asymmetry on catanionic mixtures of hydrogenated and fluorinated surfactants. Journal of Colloid and Interface Science, 2010, 341 (2), 261-266.

[67] Ruso, J. M.; Attwood, D.; Taboada, P.; Mosquera, V., Selfassociation of $\mathrm{n}$-hexyltrimethyl-ammonium bromide in aqueous electrolyte solution. Colloid Polym Sci, 2002, 280 (4), 336-341.

[68] La Mesa, C., Dependence of critical micelle concentrations on intensive variables: a reduced variables analysis. The Journal of Physical Chemistry, 1990, 94 (1), 323-326.

[69] Blanco, E.; González-Pérez, A.; Ruso, J. M.; Pedrido, R.; Prieto, G.; Sarmiento, F., A comparative study of the physicochemical properties of perfluorinated and hydrogenated amphiphiles. Journal of Colloid and Interface Science, 2005, 288 (1), 247-260.

[70] Muller, N., Temperature dependence of critical micelle concentrations and heat capacities of micellization for ionic surfactants. Langmuir, 1993, 9 (1), 96-100.

[71] Kang, K.-H.; Kim, H.-U.; Lim, K.-H., Effect of temperature on critical micelle concentration and thermodynamic potentials of micellization of anionic ammonium dodecyl sulfate and cationic octadecyl trimethyl ammonium chloride. Colloids and Surfaces A: Physicochemical and Engineering Aspects, 2001, 189 (1-3), 113121.

[72] Martínez-Landeira, P.; González-Pérez, A.; Ruso, J. M.; Prieto, G.; Sarmiento, F., Colloidal properties of benzylpenicillin: Comparison with structurally-related penicillins. Colloids and Surfaces A: Physicochemical and Engineering Aspects, 2004, 236 (1-3), 121131.

[73] Rodríguez, J. R.; González-Pérez, A.; Del Castillo, J. L.; Czapkiewicz, J., Thermodynamics of Micellization of Alkyldimethylbenzylammonium Chlorides in Aqueous Solutions. Journal of Colloid and Interface Science, 2002, 250 (2), 438-443.

[74] González-Pérez, A.; Prieto, G.; Ruso, J. M.; Sarmiento, F., Thermodynamics of self-assembly of sodium octanoate: comparison with a fully fluorinated counterpart. Molecular Physics, 2003, 101 (21), 3185-3195.

[75] González-Pérez, A.; Czapkiewicz, J.; Castillo, J. L.; Rodríguez, J. R., Micellar properties of tetradecyltrimethylammonium nitrate in aqueous solutions at various temperatures and in water-benzyl alcohol mixtures at $25^{\circ} \mathrm{C}$. Colloid Polym Sci, 2004, 282 (12), 1359-1364.

[76] Seoane, L.; Martínez-Landeira, P.; Besada, L.; Ruso, J.; Sarmiento, F.; Prieto, G., A thermodynamic study of the aggregation process of oxacillin sodium salt in aqueous solution. Colloid Polym Sci, 2002, 280 (7), 624-629.

[77] Zieliński, R.; Ikeda, S.; Nomura, H.; Kato, S., Effect of temperature on micelle formation in aqueous solutions of alkyltrimethylammonium bromides. Journal of Colloid and Interface Science, 1989, 129 (1), 175-184.

[78] Lumry, R.; Rajender, S., Enthalpy-entropy compensation phenomena in water solutions of proteins and small molecules: A 
ubiquitous properly of water. Biopolymers, 1970, 9 (10), 11251227.

[79] (a) Sugihara, G.; Hisatomi, M., Enthalpy-Entropy Compensation Phenomenon Observed for Different Surfactants in Aqueous Solution. Journal of Colloid and Interface Science, 1999, 219 (1), 31-36; (b) Sugihara, G.; Nakano, T.-Y.; Sulthana, S. B.; Rakshit, A. K., Enthalpy-Entropy Compensation Rule and the Compensation Temperature Observed in Micelle Formation of Different Surfactants in Water. What is the so-called Compensation Temperature? Journal of Oleo Science, 2001, 50 (1), 29-39.

[80] (a) Hassan, N.; Maldonado-Valderrama, J.; Gunning, A. P.; Morris, V. J.; Ruso, J. M., Investigating the effect of an arterial hypertension drug on the structural properties of plasma protein. Colloids and Surfaces B: Biointerfaces, 2011, 87 (2), 489-497; (b) Hassan, N.; Messina, P. V.; Dodero, V. I.; Ruso, J. M., Rheological properties of ovalbumin hydrogels as affected by surfactants addition. International Journal of Biological Macromolecules, 2011, 48 (3), 495-500; (c) Hassan, N.; Barbosa, L. R. S.; Itri, R.; Ruso, J. M., Fibrinogen stability under surfactant interaction. Journal of Colloid and Interface Science, 2011, 362 (1), 118-126; (d) Hassan, N.; Maldonado-Valderrama, J.; Gunning, A. P.; Morris, V. J.; Ruso, J. M., Surface Characterization and AFM Imaging of Mixed Fibrinogen-Surfactant Films. The Journal of Physical Chemistry B, 2011, 115 (19), 6304-6311; (e) Verdes, P. V.; Blanco, E.; Ruso, J. M.; Prieto, G.; Sarmiento, F., A study on the protein concentration dependence of the thermodynamics of micellization. The Journal of Chemical Thermodynamics, 2008, 40 (9), 14451450; (f) Hassan, N.; Ruso, J. M.; Somasundaran, P., Mechanisms of fibrinogen-acebutolol interactions: Insights from DSC, CD and LS. Colloids and Surfaces B: Biointerfaces, 2011, 82 (2), 581-587.

[81] Aloy, P.; Russell, R. B., Structural systems biology: modelling protein interactions. Nat Rev Mol Cell Biol, 2006, 7 (3), 188-197.

[82] (a) Lee, K.-B.; Park, S.-J.; Mirkin, C. A.; Smith, J. C.; Mrksich, M., Protein Nanoarrays Generated By Dip-Pen Nanolithography. Science, 2002, 295 (5560), 1702-1705; (b) Ruso, J. M.; Deo, N.; Somasundaran, P., Complexation between Dodecyl Sulfate Surfactant and Zein Protein in Solution. Langmuir, 2004, 20 (21), 8988-8991; (c) Ruso, J. M.; Taboada, P.; Varela, L. M.; Attwood, D.; Mosquera, V. c., Adsorption of an amphiphilic penicillin onto human serum albumin: characterisation of the complex. Biophysical Chemistry, 2001, 92 (1-2), 141-153.

[83] Taboada, P.; Mosquera, V.; Ruso, J. M.; Sarmiento, F.; Jones, M. $\mathrm{N}$., Interaction between penicillins and human serum albumin: a $\zeta$ potential study. Langmuir, 2000, 16 (17), 6795-6800.

[84] González-Pérez, A.; Ruso, J. M.; Prieto, G.; Sarmiento, F., Physicochemical study of ovalbumin in the presence of sodium dodecyl sulphate in aqueous media. Colloid Polym Sci, 2004, 282 (4), 351-356.

[85] (a) Blanco, E.; Ruso, J. M.; Prieto, G.; Sarmiento, F., Different Thermal Unfolding Pathways of Catalase in the Presence of Cationic Surfactants. The Journal of Physical Chemistry B, 2007, 111 (8), 2113-2118; (b) Ruso, J. M.; Sarmiento, F., The interaction between n-alkyl trimethylammonium bromides with poly(laspartate): a thermodynamics study. Colloid Polym Sci, 2000, 278 (8), 800-804.

[86] (a) Ruso, J. M.; González-Pérez, A.; Prieto, G.; Sarmiento, F., Study of the interactions between lysozyme and a fully-fluorinated surfactant in aqueous solution at different surfactant-protein ratios. International Journal of Biological Macromolecules, 2003, 33 (13), 67-73; (b) Blanco, E.; Messina, P.; Ruso, J. M.; Prieto, G.; Sarmiento, F., Regarding the Effect that Different Hydrocarbon/Fluorocarbon Surfactant Mixtures Have on Their Complexation with HSA. The Journal of Physical Chemistry B, 2006, 110 (23), 11369-11376; (c) Blanco, E.; Ruso, J. M.; Prieto, G.; Sarmiento, F., On relationships between surfactant type and globular proteins interactions in solution. Journal of Colloid and Interface Science, 2007, 316 (1), 37-42.

[87] Blanco, E.; Ruso, J. M.; Prieto, G.; Sarmiento, F., Electrophoretic and spectroscopic characterization of the protein patterns formed in different surfactant solutions. International Journal of Biological Macromolecules, 2008, 42 (1), 22-26.

[88] Ruso, J. M.; Attwood, D.; Garcia, M.; Taboada, P.; Varela, L. M.; Mosquera, V., A study of the interaction of the amphiphilic penicillins cloxacillin and dicloxacillin with human serum albumin in aqueous solution. Langmuir, 2001, 17 (17), 5189-5195.
[89] Ruso, J. M.; Taboada, P.; Varela, L. M.; Attwood, D.; Mosquera, V., Adsorption of an amphiphilic penicillin onto human serum albumin: Characterisation of the complex. Biophysical Chemistry, 2001, 92 (1-2), 141-153.

[90] Pérez-Rodríguez, M.; Attwood, D.; Ruso, J. M.; Taboada, P.; Varela, L. M.; Mosquera, V., Adsorption of a cationic amphiphilic drug on human serum albumin: Characterization of the complex. Physical Chemistry Chemical Physics, 2001, 3 (9), 1655-1660.

[91] Ruso, J. M.; Taboada, P.; Martínez-Landeira, P.; Prieto, G.; Sarmiento, F., A comparative study of the interaction between nafcillin and catalase by equilibrium dialysis and $\zeta$-potential measurements. Journal of Physical Chemistry B, 2001, 105 (13), 2644-2648.

[92] Taboada, P.; Mosquera, V.; Ruso, J. M.; Sarmiento, F.; Jones, M. N., Interaction between penicillins and human serum albumin: a thermodynamic study of micellar-like clusters on a protein. Langmuir, 2000, 16 (3), 934-938.

[93] He, X. M.; Carter, D. C., Atomic structure and chemistry of human serum albumin. Nature, 1992, 358 (6383), 209-215.

[94] (a) Ruso, J. M.; Gonzáez-Pérez, A.; Prieto, G.; Sarmiento, F., Study of the interaction between lysozyme and sodium octanoate in aqueous solutions. Colloids and Surfaces A: Physicochemical and Engineering Aspects, 2004, 249 (1-3), 45-50; (b) Blanco, E.; Ruso, J. M.; Sabín, J.; Prieto, G.; Sarmiento, F., Thermal stability of lysozyme and myoglobin in the presence of anionic surfactants. $J$ Therm Anal Calorim, 2007, 87 (1), 211-215.

[95] Barbosa, S.; Taboada, P.; Ruso, J. M.; Attwood, D.; Mosquera, V., Complexes of penicillins and human serum albumin studied by static light scattering. Colloids and Surfaces A: Physicochemical and Engineering Aspects, 2003, 224 (1-3), 251-256.

[96] (a) Varela, L. M.; Garcia, M.; Perez-Rodriguez, M.; Taboada, P.; Ruso, J. M.; Mosquera, V., Multilayer adsorption model for the protein-ligand interaction. Journal of Chemical Physics, 2001, 114 (17), 7682-7687; (b) Varela, L. M.; Pérez-rodríguez, M.; García, M., Cooperative binding of drugs on human serum albumin. Molecular Physics: An International Journal at the Interface Between Chemistry and Physics, 2004, 102 (1), 79 - 84.

[97] (a) Sanchez-Ruiz, J. M.; Lopez-Lacomba, J. L.; Cortijo, M.; Mateo, P. L., Differential scanning calorimetry of the irreversible thermal denaturation of thermolysin. Biochemistry, 1988, 27 (5), 16481652; (b) Layton, C. J.; Hellinga, H. W., Thermodynamic Analysis of Ligand-Induced Changes in Protein Thermal Unfolding Applied to High-Throughput Determination of Ligand Affinities with Extrinsic Fluorescent Dyes. Biochemistry, 2010, 49 (51), 1083110841.

[98] Weber, P. C.; Salemme, F. R., Applications of calorimetric methods to drug discovery and the study of protein interactions Current Opinion in Structural Biology, 2003, 13 (1), 115-121.

[99] Cerchiara, T.; Bigucci, F.; Corace, G.; Zecchi, V.; Luppi, B., Eudragit-coated albumin nanospheres carrying inclusion complexes for oral administration of indomethacin. J Incl Phenom Macrocycl Chem, 2011, 1-8.

[100] Bellavia, G.; Giuffrida, S.; Cottone, G.; Cupane, A.; Cordone, L., Protein Thermal Denaturation and Matrix Glass Transition in Different Protein-Trehalose-Water Systems. The Journal of Physical Chemistry B, 2011, 115 (19), 6340-6346.

[101] Ducry, L.; Stump, B., Antibody-Drug Conjugates: Linking Cytotoxic Payloads to Monoclonal Antibodies. Bioconjugate Chem., 2009, 21 (1), 5-13.

[102] Hassan, N.; Verdes, P. V.; Ruso, J. M., Assessment of interactions between four proteins and benzothiazole derivatives by DSC and CD. The Journal of Chemical Thermodynamics, 2011, 43 (3), 399404.

[103] Hassan, N.; Ruso, J. M.; Somasundaran, P., Mechanisms of fibrinogen-acebutolol interactions: Insights from DSC, CD and LS Colloids and Surfaces B: Biointerfaces, 2011, 82 (2), 581-587.

[104] Alderson, R. G.; De Ferrari, L.; Mavridis, L.; McDonagh, J. L.; Mitchell, J. B.; Nath, N., Enzyme informatics. In Curr Top Med Chem, Netherlands, 2012; Vol. 12, pp 1911-23.

[105] Yang, F.; Zhou, B.-R.; Zhang, P.; Zhao, Y.-F.; Chen, J.; Liang, Y., Binding of ferulic acid to cytochrome $\mathrm{c}$ enhances stability of the protein at physiological $\mathrm{pH}$ and inhibits cytochrome c-induced apoptosis. Chemico-Biological Interactions, 2007, 170 (3), 231243.

[106] Leiros, H.-K. S.; Brandsdal, B. O.; McSweeney, S. M., Biophysical characterization and mutational analysis of the antibiotic resistance 
protein NimA from Deinococcus radiodurans. Biochimica et Biophysica Acta (BBA) - Proteins \& Proteomics, 2010, 1804 (4), 967-976.

[107] Giannetti, A. M.; Wong, H.; Dijkgraaf, G. J. P.; Dueber, E. C.; Ortwine, D. F.; Bravo, B. J.; Gould, S. E.; Plise, E. G.; Lum, B. L.; Malhi, V.; Graham, R. A., Identification, Characterization, and Implications of Species-Dependent Plasma Protein Binding for the
Oral Hedgehog Pathway Inhibitor Vismodegib (GDC-0449). Journal of Medicinal Chemistry, 2011, 54 (8), 2592-2601.

[108] Plantinga, A.; Witte, A.; Li, M.-H.; Harmon, A.; Choi, S. K.; Banaszak Holl, M. M.; Orr, B. G.; Baker, J. R.; Sinniah, K., Bioanalytical Screening of Riboflavin Antagonists for Targeted Drug Delivery-A Thermodynamic and Kinetic Study. ACS Medicinal Chemistry Letters, 2011, 2 (5), 363-367. 